\title{
Efficacité et innocuité des corticostéroïdes dans le traitement de la COVID-19 selon des données pour la COVID-19, d'autres infections aux coronavirus, l'influenza, la pneumonie extrahospitalière et le syndrome de détresse respiratoire aiguë : revue systématique et méta-analyse
}

\author{
Zhikang Ye MPharm, Ying Wang MPharm, Luis Enrique Colunga-Lozano MD MSc, Manya Prasad MD MBBS, \\ Wimonchat Tangamornsuksan PharmD PhD, Bram Rochwerg MD MSc, Liang Yao MSc, Shahrzad Motaghi MSc, \\ Rachel J. Couban MA MISt, Maryam Ghadimi PharmD BCPS, Malgorzata M. Bala MD PhD, Huda Gomaa MSc, \\ Fang Fang MD, Yingqi Xiao MN, Gordon H. Guyatt MD MSc
}

Citation : CMAJ 2020 July 6;192:E756-67. doi: 10.1503/cmaj.200645-f; diffusion hâtive le 14 mai 2020

Voir la version anglaise de l'article ici : www.cmaj.ca/lookup/doi/10.1503/cmaj.200645; voir un article connexe (en anglais) ici : www.cmaj.ca/lookup/doi/10.1503/cmaj.200648

\section{RÉSUMÉ}

CONTEXTE : Il existe très peu de données directes sur l'administration de corticostéroïdes aux patients atteints de la maladie à coronavirus 2019 (COVID-19). Les données indirectes sur des maladies associées devront donc guider les conclusions quant aux bénéfices et aux préjudices associés à cette pratique. Dans le but d'appuyer la rédaction d'une ligne directrice sur la prise en charge de la COVID-19, nous avons réalisé des revues systématiques sur les effets des corticostéroïdes dans le traitement de la COVID-19 et de maladies respiratoires aiguës sévères associées.

MÉTHODES : Dans des bases de données biomédicales chinoises et internationales et des sources de prépublications, nous avons cherché les essais randomisés et contrôlés (ERC) et les études d'observation comparant des patients atteints de la COVID-19, du syndrome respiratoire aigu sévère (SRAS) ou du syndrome respiratoire du Moyen-Orient (SRMO) ayant reçu des corticostéroïdes à des patients semblables n'ayant pas reçu ce type de médicaments. Pour le syndrome de détresse respiratoire aiguë (SDRA), l'influenza et la pneumonie extrahospitalière $(\mathrm{PEH})$, nous avons mis à jour les revues systématiques rigoureuses les plus récentes. Nous avons réalisé des méta-analyses à effets aléatoires pour cerner les risques relatifs, puis nous avons utilisé le risque de référence des patients atteints de la COVID-19 pour calculer les effets absolus.

RÉSULTATS : Pour le SDRA, selon 1 petite étude de cohorte sur des patients atteints de la COVID-19 et 7 ERC sur des patients atteints d'une autre maladie (risque relatif : 0,72 , intervalle de confiance $[I C]$ de $95 \% 0,55-0,93$, différence entre les moyennes [DM] 17,3\% plus faible, données de faible qualité), les corticostéroïdes pourraient réduire le risque de mortalité. Chez les patients atteints d'une forme grave de COVID-19 sans SDRA, 2 études d'observation ont généré des données directes de très faible qualité montrant une augmentation du risque de mortalité avec l'administration de corticostéroïdes (rapport de risques 2,30, IC de $95 \%$ 1,00-5,29, DM 11,9\% plus élevé). C'est aussi le cas de données observationnelles sur l'influenza. Des données observationnelles de très faible qualité sur le SRAS et le SRMO montrent peu ou pas de réduction dans le risque de mortalité. Des essais randomisés et contrôlés sur la PEH suggèrent que les corticostéroïdes pourraient réduire le risque de mortalité (risque relatif 0,70 , IC de $95 \%$ 0,50-0,98, DM 3,1\% plus faible, données de très faible qualité), et augmenter le risque d'hyperglycémie.

INTERPRÉTATION : Les corticostéroïdes pourraient réduire le risque de mortalité pour les patients atteints de la COVID-19 avec SDRA. Pour les patients atteints d'une forme grave de COVID-19 sans SDRA, les données sur les bénéfices provenant de différentes sources sont incohérentes et de très faible qualité. 
e 11 mars 2020, l'Organisation mondiale de la Santé a qualifié de pandémie la maladie à coronavirus 2019 (COVID-19) ${ }^{1}$. La propagation mondiale de la COVID-19 est une grande menace à la santé humaine.

Les médecins traitent souvent les patients atteints de la COVID19 par l'administration de corticostéroïdes ${ }^{2}$. Cette pratique est toutefois controversée : 2 publications récentes dans The Lancet ont fait état de points de vue divergents fondés en partie sur des études portant sur le syndrome respiratoire aigu sévère (SRAS), le syndrome respiratoire du Moyen-Orient (SRMO) et l'influenza. Une étude déconseillait l'administration de corticostéroïdes dans le traitement de la COVID-19, alors que l'autre la recommandait pour certains patients ${ }^{3,4}$.

La formulation de recommandations sur l'administration de corticostéroïdes aux patients atteints de la COVID-19 exige un examen systématique des données disponibles. Ainsi, pour appuyer un guide de pratique clinique sur la prise en charge des patients atteints de la COVID-195 , nous avons mené une série de revues systématiques. Comme nous anticipions un manque de données directes pour la COVID-19, nous avons inclus les données disponibles portant sur l'administration de corticostéroïdes dans le traitement du syndrome de détresse respiratoire aiguë (SDRA), du SRAS, du SRMO, de l'influenza et de la pneumonie extrahospitalière $(\mathrm{PEH})$, des sources de données indirectes pouvant nous guider dans la détermination de l'efficacité et de l'innocuité des corticostéroïdes dans le traitement de la COVID-19.

\section{Méthodes}

Pour le SDRA, nous avons utilisé les définitions des études admissibles, et celle de l'Organisation mondiale de la Santé pour les formes graves de COVID-19 (fièvre ou suspicion d'infection respiratoire, ainsi qu'un des signes suivants : fréquence respiratoire $>30$ cycles/min, intense détresse respiratoire ou saturation en oxygène $\left[\mathrm{SpO}_{2}\right] \leq 93 \%$ en air ambiant $\left.{ }^{6}\right)$.

Pour la COVID-19, le SRAS et le SRMO, nous avons inclus toutes les études primaires admissibles à nos revues systématiques. Pour le SDRA, l'influenza et la PEH, nous avons choisi les revues systématiques rigoureuses les plus récentes et les études primaires admissibles récentes. Le choix des paramètres a été encadré par notre protocole préliminaire, par les conseils du groupe de travail pour la ligne directrice et par les conclusions des études admissibles.

\section{Stratégies de recherche et critères de sélection}

L'annexe 1 (accessible en anglais au www.cmaj.ca/lookup/suppl/ doi:10.1503/cmaj.200645/-/DC1) présente le protocole établi avant d'entamer les revues systématiques, qui suit le modèle PRISMA (Preferred Reporting Items for Systematic Reviews and Meta-Analyses) ${ }^{7}$.

\section{COVID-19, SRAS et SRMO}

Avec l'aide d'une bibliothécaire médicale (R.J.C.), nous avons cherché les documents publiés dans MEDLINE, Embase, PubMed et le Cochrane Central Register of Controlled Trials du début de la couverture de ces bases de données jusqu'au 19 avril 2020, et dans
medRxiv, jusqu'au 25 avril 2020. Pour les études portant sur des patients atteints de la COVID-19, nous avons aussi cherché dans des bases de données chinoises, notamment la China National Knowledge Infrastructure (CNKI), Wanfang, Chongqing VIP Information (CQVIP) et ChinaXiv. L'annexe 2 (accessible en anglais au www.cmaj.ca/lookup/suppl/doi:10.1503/cmaj.200645/-/DC1) présente la stratégie de recherche complète.

Nous avons inclus les essais randomisés et contrôlés (ERC), les études de cohorte et les études cas-témoins comparant les patients atteints de la COVID-19, du SRAS ou du SRMO ayant reçu des corticostéroïdes à ceux qui n'en ont pas reçu. Nous n'avons inclus que les études de cohorte et les études cas-témoins qui comportaient une analyse ajustée, sauf si aucune étude ne présentait ce type d'analyse. Dans ce dernier cas, nous avons inclus les analyses non ajustées. Pour les études présentant des recoupements (utilisant les mêmes sources de données), nous n'avons inclus que l'étude la plus importante, à moins qu'une plus petite étude comprenne des analyses supplémentaires utiles.

\section{SDRA, influenza et PEH}

Nous avons réalisé des recherches séparées pour le SDRA, l'influenza et la $\mathrm{PEH}$, en utilisant un processus en 2 étapes (voir l'annexe 2 pour connaître la stratégie de recherche). Nous avons d'abord cherché à repérer les revues systématiques s'intéressant aux effets des corticostéroïdes sur les patients atteints d'un SDRA, de l'influenza ou d'une PEH dans MEDLINE, Embase, la Cochrane Database of Systematic Reviews et Epistemonikos et avons choisi la revue systématique rigoureuse la plus récente. Ensuite, nous avons cherché les études publiées après la recherche de ces revues dans MEDLINE, Embase et ClinicalTrials.gov pour le SDRA et la PEH, et dans MEDLINE, Embase, PubMed et le Cochrane Central Register of Controlled Trials pour l'influenza. Pour le SDRA et la PEH, nous n'avons inclus que des ERC. Pour l'influenza, nous avons inclus des ERC et des études de cohorte.

Pour toutes les recherches, 2 personnes ont parcouru de manière indépendante les titres et les résumés, puis le texte complet des études prometteuses pour déterminer leur admissibilité. Les désaccords ont été résolus par une discussion ou, si nécessaire, par l'opinion d'une troisième personne. Il n'y avait aucune restriction de langue.

\section{Analyse de données}

Deux personnes ont extrait de manière indépendante les paramètres des études, une troisième personne réglant les désaccords au besoin. Les paramètres comprennent le risque de mortalité, la durée du séjour aux soins intensifs, la durée du séjour à I'hôpital, la durée de la ventilation mécanique, la nécessité d'une ventilation mécanique, la détection d'acide ribonucléique (ARN) viral, l'intervalle de temps où le virus était libéré, le risque d'hyperglycémie grave, le risque de surinfection, la faiblesse neuromusculaire et les saignements gastro-intestinaux.

Nous avons calculé des estimations sommaires à l'aide de Stata ou de Review Manager, et les effets relatifs (rapports des cotes $[R C]$, risques relatifs et rapports de risque) et les intervalles de confiance (IC) de $95 \%$ pour les paramètres dichotomiques, ainsi que les différences entre moyennes (DM) et les IC de 95\% 
pour les paramètres continus à l'aide d'un modèle à effets aléatoires. Pour les paramètres continus et les estimations ajustées, nous avons utilisé la méthode de l'inverse de la variance (DerSimonian et Laird), et la méthode de Mantel-Haenszel pour les paramètres dichotomiques des ERC. Lorsqu'il y avait des incohérences entre les études, nous avons tranché en évaluant la différence entre les estimations ponctuelles et le chevauchement des intervalles de confiances, ainsi que la statistique $I^{2}$. Pour les paramètres dichotomiques, nous avons calculé les effets absolus des traitements en appliquant les effets relatifs au risque chez les patients n'ayant pas reçu de corticostéroïdes dans 2 groupes : les patients atteints d'une forme grave de COVID-19 et les patients atteints de la COVID-19 avec SDRA. Le risque de mortalité de référence pour les patients atteints de la COVID-19 et du SDRA a été déterminé grâce à une étude d'observation portant sur des patients atteints de la COVID-19 et du SDRA ${ }^{8}$, et celui pour les patients atteints d'une forme grave de COVID-19, grâce à une étude d'observation portant sur des patients atteints de COVID-19 grave $^{2}$. Pour les autres paramètres, nous nous sommes fondés sur le risque de référence de la médiane des groupes n'ayant pas reçu de corticostéroïdes dans les études incluses.

\section{Évaluation du risque de biais}

Nous avons utilisé ROBIS, un outil d'évaluation du risque de biais $^{9}$, pour choisir les revues systématiques les plus rigoureuses à mettre à jour. Nous avons utilisé une version modifiée de l'outil d'évaluation du risque de biais de Cochrane ${ }^{10}$ pour évaluer le risque de biais dans les ERC et une version révisée de l'échelle de Newcastle-Ottawa ${ }^{11,12}$ pour les études d'observation (détails accessibles au www.evidencepartners.com/ resources/methodological-resources/). Deux personnes ont évalué de manière indépendante le risque de biais, en résolvant les désaccords avec l'aide d'une troisième personne au besoin.

\section{Classement de la qualité des données}

Nous avons utilisé l'approche GRADE (Grading of Recommendations Assessment, Development and Evaluation) pour classer la qualité des données comme élevée, modérée, faible ou très faible pour chaque paramètre ${ }^{13}$. L'évaluation porte sur les éléments suivants : risque de biais ${ }^{14}$, imprécisions ${ }^{15}$, incohérences ${ }^{16}$, données indirectes ${ }^{17}$ et biais de publication ${ }^{18}$. En cas de problème important dans l'un ou l'autre de ces domaines (par exemple, pour le risque de biais), nous avons réduit la cote de qualité des données. Parce que les effets des corticostéroïdes pourraient différer pour les personnes atteintes de la COVID-19 et celles atteintes d'autres maladies, et comme nous utilisons une approche indirecte, nous avons réduit la cote de qualité des données d'un niveau pour les bénéfices chez les patients atteints du SRAS et du SRMO, et de 2 niveaux pour les patients atteints du SDRA, de l'influenza ou d'une PEH. Parce que nous avons considéré que les préjudices étaient plus susceptibles de s'appliquer à la population cible que les bénéfices, pour toutes les populations, nous avons réduit la cote de qualité des données d'un niveau.

\section{Approbation éthique}

L'approbation éthique n'avait pas à être demandée pour cette revue systématique.

\section{Résultats}

L'annexe 3 (accessible en anglais au www.cmaj.ca/lookup/suppl/ doi:10.1503/cmaj.200645/-/DC1) présente le processus de sélection des études. Notre recherche pour la COVID-19, le SRAS et le SRMO a donné 5120 résultats. Après avoir retiré les duplicata, parcouru les titres et les résumés et consulté les textes complets, nous avons finalement inclus 1 étude de cohorte ${ }^{8}$ portant sur 84 patients atteints de la COVID-19 et du SDRA, 5 études de cohorte ${ }^{19-23}$ portant sur 679 patients atteints de la COVID-19, sans SDRA, 3 études (2 études de cohorte ${ }^{24,25}$ et $1 E^{E R C^{26}}$ ) portant sur 7087 patients atteints du SRAS et 2 études de cohorte ${ }^{27,28}$ portant sur 623 patients atteints du SRMO.

Notre recherche de revues systématiques sur le SDRA a donné 836 résultats. Nous avons finalement choisi de mettre à jour une revue systématique publiée en $2019^{29}$. Notre recherche d'études primaires a permis de trouver 1 nouvel ERC admissible publié en $2020^{30}$. Avec les 6 ERC contenus dans la revue systématique, nous avons inclus $7 \mathrm{ERC}^{30-36}$ portant sur 851 patients.

Notre recherche de revues systématiques sur l'influenza a donné 525 résultats. Nous avons choisi de mettre à jour une revue systématique publiée en $2019^{37}$. Notre recherche d'études primaires a permis de trouver 1 nouvelle étude admissible publiée en $2020^{38}$. Avec les 30 études présentées dans la revue systématique, nous avons relevé 31 études admissibles ${ }^{39-69}$, parmi lesquelles 21 portant sur 9536 patients ont été incluses aux méta-analyses ${ }^{41,43-47,50,52,53,55-61,63-65,68,69}$.

Notre recherche de revues systématique sur la PEH a donné 346 résultats. Nous avons choisi de mettre à jour une revue systématique publiée en $2015^{70}$. Notre recherche d'études primaires a permis de trouver 1 nouvelle étude admissible publiée en $2016^{71}$. En incluant les 12 ERC inclus dans la revue systématique, nous nous sommes intéressés à $13 \mathrm{ERC}^{71-83}$ portant sur 2095 patients.

L'annexe 4 (accessible en anglais au www.cmaj.ca/lookup/ suppl/doi:10.1503/cmaj.200645/-/DC1) présente les paramètres des études incluses. L'annexe 5 (accessible en anglais au www. cmaj.ca/lookup/suppl/doi:10.1503/cmaj.200645/-/DC1) présente l'évaluation du risque de biais pour chaque étude. Des graphiques en forêts des résultats des méta-analyses pour le risque de mortalité sont présentés aux figures 1 à 5, et, pour les autres paramètres, à l'annexe 6 (accessible en anglais au www.cmaj.ca/ lookup/suppl/doi:10.1503/cmaj.200645/-/DC1).

\section{SDRA}

Les données pour les patients atteints de la COVID-19 et d'un SDRA étaient disponibles dans 1 étude d'observation portant sur 84 patients $^{8}$. Elles suggéraient que l'administration de corticostéroïdes pourrait grandement réduire le risque de mortalité comparativement à l'absence de ce médicament (rapport de risque : 0,41 , IC de $95 \%$ 0,20-0,83, DM 29,2\% plus faible, données de très faible qualité) (tableau 1).

Les données pour les patients atteints du SDRA sans COVID-19 provenaient de $7 \mathrm{ERC}^{30-36}$ portant sur 851 patients (tableau 2). Nous avons considéré que les données pour la plupart des paramètres étaient de qualité élevée pour le SDRA. Après avoir réduit de 2 niveaux la cote de qualité des données en raison des 


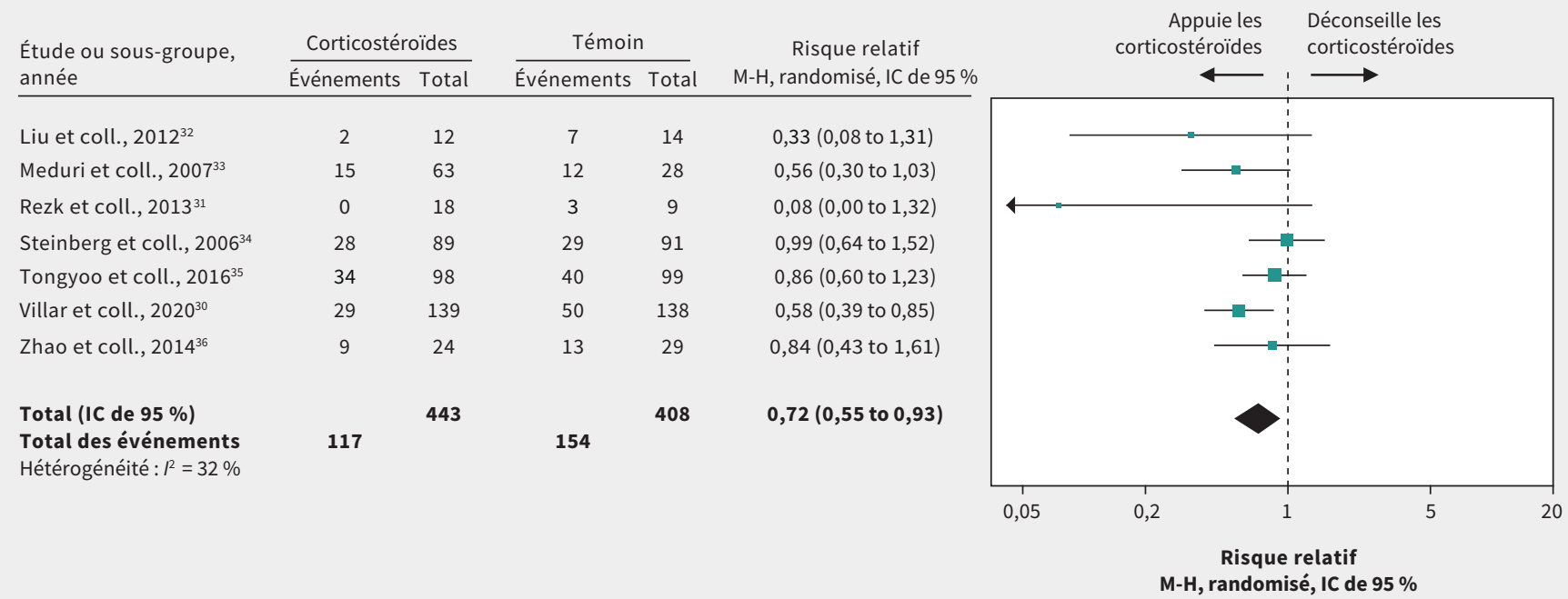

Figure 1 : Effet des corticostéroïdes sur le risque de mortalité chez les patients atteints du syndrome de détresse respiratoire aiguë sans maladie à coronavirus 2019. Remarque : $\mathrm{IC}=$ intervalle de confiance, $\mathrm{M}-\mathrm{H}=$ Mantel-Haenszel.

\begin{tabular}{|c|c|c|c|c|}
\hline Étude, année & HR (IC de $95 \%)$ & Pondération (\%) & $\begin{array}{r}\text { Appuie les } \\
\text { corticostéroïdes }\end{array}$ & $\begin{array}{l}\text { Déconseille les } \\
\text { corticostéroïdes } \\
\longrightarrow\end{array}$ \\
\hline Li et coll., $2020^{19}$ & $2,12(0,78$ to 5,76$)$ & 69,23 & & \\
\hline Lu et coll., $2020^{23}$ & $2,78(0,96$ to 19,26$)$ & 30,77 & & $\rightarrow$ \\
\hline \multirow[t]{3}{*}{ Total $\left(I^{2}=0,0 \%, p=0,768\right)$} & $2,30(1,00$ to 5,29$)$ & 100,00 & & \\
\hline & & & 0,0519 & 19,3 \\
\hline & & & $\begin{array}{r}\text { Rappc } \\
\text { IV, rando }\end{array}$ & $\begin{array}{l}\text { risques } \\
\text { IC de } 95 \%\end{array}$ \\
\hline
\end{tabular}

Figure 2 : Effet des corticostéroïdes sur le risque de mortalité chez les patients atteints de la forme grave de la maladie à coronavirus 2019 . La pondération provient des analyses à effets aléatoires. Remarque : IC = intervalle de confiance, $\mathrm{HR}=$ rapport de risques (Hazard Ratio), IV = inverse de la variance.

\begin{tabular}{|c|c|c|c|c|}
\hline Étude, année & HR (IC de $95 \%)$ & Pondération (\%) & $\begin{array}{r}\text { Appuie les } \\
\text { corticostéroïdes }\end{array}$ & $\begin{array}{l}\text { Déconseille les } \\
\text { corticostéroïdes } \\
\longrightarrow\end{array}$ \\
\hline Long et coll., $2016^{25}$ & $0,62(0,4$ to 0,96$)$ & 60,96 & & \\
\hline Lau et coll., $2009^{24}$ & $1,29(0,55$ to 2,70$)$ & 39,04 & & - \\
\hline \multirow[t]{2}{*}{ Total $\left(P^{2}=60,5 \%, p=0,111\right)$} & $0,83(0,41$ to 1,66$)$ & 100,00 & & $\longrightarrow$ \\
\hline & & & $\begin{array}{r}\text { Rappo } \\
\text { IV, randor }\end{array}$ & $\begin{array}{l}\text { risques } \\
\text { IC de } 95 \%\end{array}$ \\
\hline
\end{tabular}

Figure 3 : Effet des corticostéroïdes sur le risque de mortalité chez les patients atteints du syndrome respiratoire aigu sévère. La pondération provient des analyses à effets aléatoires. Remarque : IC = intervalle de confiance, HR = rapport de risques (Hazard Ratio), IV = inverse de la variance. 


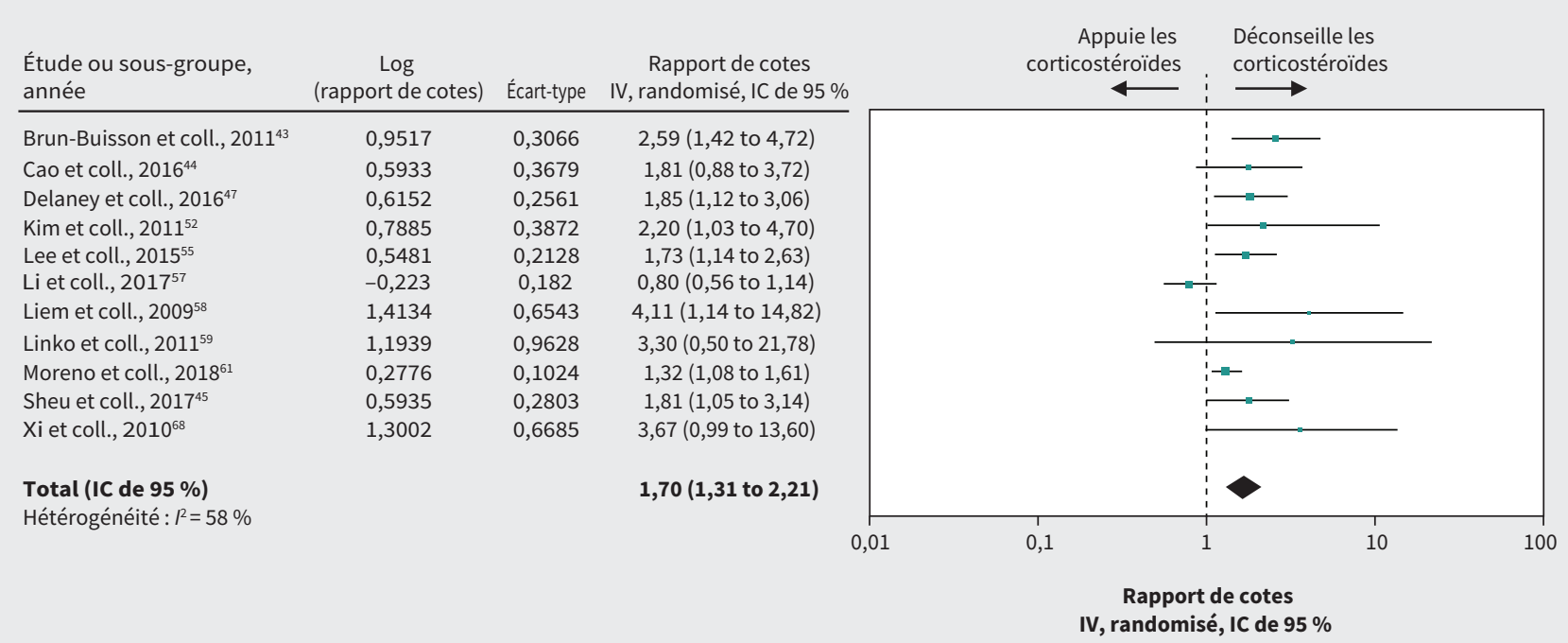

Figure 4 : Effet des corticostéroïdes sur le risque de mortalité chez les patients atteints d'influenza. Remarque : IC = intervalle de confiance, IV = inverse de la variance.

\begin{tabular}{|c|c|c|c|c|c|c|c|c|c|}
\hline \multirow{2}{*}{$\begin{array}{l}\text { Étude ou sous-groupe, } \\
\text { année }\end{array}$} & \multicolumn{2}{|c|}{ Corticostéroïdes } & \multicolumn{2}{|c|}{ Soins habituels } & \multirow{2}{*}{$\begin{array}{c}\text { Risque relatif } \\
\mathrm{M}-\mathrm{H} \text {, randomisé, IC de } 95 \%\end{array}$} & & \multirow{2}{*}{$\begin{array}{r}\text { Appuie les } \\
\text { corticostéroïdes }\end{array}$} & \multirow{2}{*}{$\begin{array}{l}\text { Déconseille les } \\
\text { corticostéroïdes } \\
\longrightarrow\end{array}$} & \\
\hline & Événements & $\overline{\text { Total }}$ & Événements & $\overline{\text { Total }}$ & & & & & \\
\hline \multicolumn{10}{|l|}{ Grave } \\
\hline Confalonieri et coll., $2005^{73}$ & 0 & 23 & 8 & 23 & $0,06(0,00$ to 0,96$)$ & $\longleftarrow$ & & & \\
\hline El-Ghamrawy et coll., $2006^{74}$ & 3 & 17 & 6 & 17 & $0,50(0,15$ to 1,68$)$ & & & & \\
\hline Gang et coll., $2016^{71}$ & 3 & 29 & 3 & 29 & $1,00(0,22$ to 4,55$)$ & & & & \\
\hline Marik et coll., $1993^{76}$ & 1 & 14 & 3 & 16 & $0,38(0,04$ to 3,26$)$ & & & & \\
\hline Nafae et coll., $2013^{79}$ & 4 & 60 & 6 & 20 & $0,22(0,07$ to 0,71$)$ & & & & \\
\hline Sabry et coll., $2011^{43}$ & 2 & 40 & 6 & 40 & $0,33(0,07$ to 1,55$)$ & & & & \\
\hline Torres et coll., $2015^{82}$ & 6 & 61 & 9 & 59 & $0,64(0,24$ to 1,70$)$ & & & & \\
\hline Sous-total (IC de $95 \%$ ) & & 244 & & 204 & $0,43(0,26$ to 0,73$)$ & & & & \\
\hline $\begin{array}{l}\text { Total des événements } \\
\text { Hétérogénéité : } P^{2}=0 \%\end{array}$ & 19 & & 41 & & & & & & \\
\hline \multicolumn{10}{|l|}{ Moins grave } \\
\hline Blum et coll., $2015^{72}$ & 16 & 392 & 13 & 393 & $1,23(0,60$ to 2,53$)$ & & & & \\
\hline Fernández-Serrano et coll., $2011^{75}$ & 1 & 23 & 1 & 22 & $0,96(0,06$ to 14,37$)$ & & & & \\
\hline McHardy et coll., $1972^{77}$ & 3 & 40 & 9 & 86 & $0,72(0,20$ to 2,51$)$ & & & & \\
\hline Meijvis et coll., $2011^{78}$ & 9 & 151 & 11 & 153 & $0,83(0,35$ to 1,94$)$ & & & & \\
\hline Snijders et coll., $2010^{81}$ & 6 & 104 & 6 & 109 & $1,05(0,35$ to 3,15$)$ & & & & \\
\hline Wagner et coll., $1956^{83}$ & 1 & 52 & 1 & 61 & $1,17(0,08$ to 18,30$)$ & & & & \\
\hline Sous-total (IC de $95 \%$ ) & & 762 & & 824 & $1,00(0,64$ to 1,56$)$ & & & & \\
\hline $\begin{array}{l}\text { Total des événements } \\
\text { Hétérogénéité }: P^{2}=0 \%\end{array}$ & 36 & & 41 & & & & & & \\
\hline \multirow{3}{*}{$\begin{array}{l}\text { Total (IC de } 95 \% \text { ) } \\
\text { Total des événements } \\
\text { Hétérogénéité : } P^{2}=0 \%\end{array}$} & & 1006 & & 1028 & $0,70(0,50$ to 0,98$)$ & & & & \\
\hline & 55 & & 82 & & & & & & \\
\hline & & & & & & 0,01 & 0,1 & $\begin{array}{l}1 \\
10\end{array}$ & 100 \\
\hline
\end{tabular}

Figure 5 : Effet des corticostéroïdes sur le risque de mortalité chez les patients atteints d'une pneumonie extrahospitalière. Remarque : IC = intervalle de confiance, $\mathrm{M}-\mathrm{H}=$ Mantel-Haenszel. 
données indirectes, elles étaient de faible qualité pour la COVID-19. Ces ERC semblent indiquer que les corticostéroïdes pourraient réduire de manière importante le risque de mortalité (risque relatif 0,72, IC de 95\% 0,55-0,93, DM 17,3\% plus faible, données de faible qualité) (figure 1). La très faible qualité des données soulève la possibilité que les corticostéroïdes auraient peu ou pas d'effets sur la durée du séjour aux soins intensifs ${ }^{32-34}$ (DM 0,1 jour de plus, IC de $95 \% 3,0$ jours de moins à 3,2 jours de plus), mais pourraient réduire la durée du séjour à l'hôpital ${ }^{33,34,36}$ (DM 3,6 jours de moins, IC de 95\% 0,02-7,2 jours de moins). Des données de faible qualité montrent que l'administration de corticostéroïdes pourrait réduire la durée de la ventilation mécanique (DM -4,8 jours, IC de $95 \%-7,0$ à $-2,6)^{30,31,33-36}$, mais augmenter le risque d'hyperglycémie grave (augmentation du risque de $8,1 \%$, IC de $95 \% 0,7 \%-16,2 \%)^{30,33,35}$, avec peu ou pas d'effets négatifs sur la faiblesse neuromusculaire ${ }^{33,34}$, les saignements gastro-intestinaux $x^{35,36}$ et le risque de surinfection ${ }^{30,33-36}$.

\section{Forme grave de COVID-19 : données directes d'études d'observation}

Des données de très faible qualité de 2 études de cohorte ${ }^{19,23}$ portant sur 331 patients atteints d'une forme grave de COVID-19 ont soulevé la possibilité que les corticostéroïdes augmentent le risque de mortalité comparativement à la non-administration de ce médicament (rapport de risques 2,30, IC de 95\% 1,00-5,29, DM $11,9 \%$ plus élevé) (tableau 3, figure 2). Une étude de cohorte ${ }^{20} \mathrm{a}$ rapporté une augmentation du risque de mortalité, toutes populations confondues ou de l'admission aux soins intensifs des patients à qui on administre des corticostéroïdes. De plus, 2 études de cohorte ${ }^{21,22}$ ont suggéré que l'administration de corticostéroïdes serait associée à une période plus longue avant la clairance du virus (données de très faible qualité).

Forme grave de COVID-19 : données indirectes d'études d'observation et d'un essai randomisé sur des patients atteints du SRAS

Deux études de cohorte ${ }^{24,25}$ portant sur 6129 patients atteints du SRAS ont fourni des données de faible qualité sur l'effet des corticostéroïdes sur le risque de mortalité; la cote de qualité des données est encore moindre avec l'ajustement en raison des données indirectes (rapport de risques 0,83, IC de $95 \%$ 0,41-1,66, données de très faible qualité) (tableau 4, figure 3 ). Un ERC ${ }^{26}$ pour lequel on a étudié 16 patients atteints du SRAS traités à la ribavirine ayant reçu ou non des corticostéroïdes a soulevé la possibilité qu'un traitement précoce ( $<7$ jours de maladie) à l'hydrocortisone augmenterait la durée médiane avant laquelle l'ARN du coronavirus associé au SRAS (SRAS-CoV) est indétectable dans le plasma (DM 4,0 jours de plus, IC de 95\% 2,0-6,0 jours, données de très faible qualité pour le SRAS, avec les ajustements en raison des données indirectes) (tableau 4).

\section{Forme grave de COVID-19: données indirectes d'études d'observation sur des patients atteints du SRMO}

Une étude de cohorte ${ }^{28}$ portant sur 290 patients atteints du SRMO suggère une possible réduction du risque de mortalité associée à l'administration de corticostéroïdes (rapport de cotes 0,75 , IC de $95 \%$ 0,52-1,07, données de très faible qualité pour le SRMO, avec considérations supplémentaires en raison des données indirectes) (tableau 5). Les données de 189 patients de la même étude ${ }^{28}$ suggèrent que l'administration de corticostéroïdes pourrait être associée à un retard dans la clairance de I'ARN du coronavirus responsable du syndrome respiratoire du Moyen-Orient (CoV-SRMO) (rapport de risques 0,35, IC de $95 \%$ $0,17-0,72$, données de très faible qualité pour le SRMO, avec les considérations supplémentaires en raison des données indirectes) (tableau 5).

Forme grave de COVID-19 : données indirectes d'études d'observation sur des patients atteints de l'influenza Les données pour l'influenza provenant de 11 études de cohorte ${ }^{43-45,47,52,55,57-59,61,68}$ portant sur 8530 patients avec des estimations ajustées pour le risque de mortalité suggèrent que les corticostéroïdes pourraient accroître le risque de mortalité (rapport de cotes 1,70, IC de $95 \% 1,31-2,21$, DM 6,1\% plus élevé, données de faible qualité pour l'influenza et considérées comme très faible pour la COVID-19 en raison des données indirectes) (tableau 6, figure 4). Des données de très faible qualité

Tableau 1 : Résumé des conclusions et cote GRADE : effets des corticostéroïdes chez les patients atteints de la COVID-19 et du SDRA, selon les données directes d'études d'observation sur des patients atteints de la COVID-19 et du SDRA

\begin{tabular}{|c|c|c|c|c|c|}
\hline \multirow[b]{2}{*}{ Résultats } & \multirow[b]{2}{*}{ Effets relatifs } & \multicolumn{2}{|c|}{ Estimations des effets absolus } & \multirow[b]{2}{*}{$\begin{array}{l}\text { Qualité des } \\
\text { données probantes }\end{array}$} & \multirow[b]{2}{*}{$\begin{array}{l}\text { Résumé en langage } \\
\text { simple }\end{array}$} \\
\hline & & $\begin{array}{c}\text { Risque de référence } \\
\text { du groupe témoin* } \\
(\%)\end{array}$ & $\begin{array}{c}\text { Écart } \\
\text { (IC de } 95 \%) \\
(\%)\end{array}$ & & \\
\hline $\begin{array}{l}\text { Risque de } \\
\text { mortalité }\end{array}$ & $\begin{array}{l}\text { HR 0,41 (IC de } 95 \% \text { 0,20-0,83) } \\
\text { Selon les données d'une étude } \\
\text { d'observation }{ }^{8} \text { portant sur } \\
84 \text { patients atteints de la } \\
\text { COVID-19 et du SDRA }\end{array}$ & 61,8 & $\begin{array}{c}-29,2 \\
(-44,3 \text { à }-6,8)\end{array}$ & $\begin{array}{l}\text { Très faible } \\
\text { (imprécision } \\
\text { importantet) }\end{array}$ & $\begin{array}{l}\text { Les effets des } \\
\text { corticostéroïdes sur } \\
\text { le risque de mortalité } \\
\text { sont très incertains. }\end{array}$ \\
\hline $\begin{array}{l}\text { Remarque : } \mathrm{C} \\
\text { intervalle de } \\
\text { ^Risque de } \mathrm{m} \\
\text { †Étude d'obse } \\
\text { qualité pour i }\end{array}$ & $\begin{array}{l}\text { aladie à coronavirus } 2019, \text { GRADE = Gra } \\
\text { DRA = syndrome de détresse respiratoir } \\
\text { f́férence pour les patients atteints de la } \\
\text { données de faible qualité. Bien que l'IC }\end{array}$ & $\begin{array}{l}\text { f Recommendations Assessn } \\
\text { ë. } \\
\text {-19 avec SDRA n'ayant pas } \\
\text { le restreint, la petite taille d }\end{array}$ & $\begin{array}{l}\text { Development anc } \\
\text { de corticostéroïde } \\
\text { hantillon et le gr }\end{array}$ & $\begin{array}{l}\text { Iuation, } \mathrm{HR}=\text { rapport de ris } \\
\text { ffet peu plausible ont men }\end{array}$ & $\begin{array}{l}\text { s (Hazard Ratio), IC = } \\
\text { retrait d'un niveau de }\end{array}$ \\
\hline
\end{tabular}


pour l'influenza avec les considérations supplémentaires en raison des données indirectes qui proviennent d'études de cohorte ne comportant pas d'analyses ajustées ont soulevé la possibilité que les corticostéroïdes augmentent le risque de surinfection (rapport de cotes 2,74, IC de $95 \% 1,51-$ $4,95)^{43,44,47,52,55,57,65}$ et le nombre de patients nécessitant une ventilation mécanique (rapport de cotes 5,54, IC de $95 \% 1,83-$ $16,80)^{52,57,59,61}$ (tableau 6).

Tableau 2 : Résumé des conclusions et cote GRADE : effets des corticostéroïdes chez les patients atteints de la COVID-19 et d'un SDRA, selon les données indirectes d'essais randomisés et contrôlés sur des patients atteints d'un SDRA sans COVID-19

Estimations des effets absolus

\begin{tabular}{|c|c|}
\hline Résultats & Effets relatifs \\
\hline $\begin{array}{l}\text { Risque de } \\
\text { mortalité }\end{array}$ & $\begin{array}{l}\text { RR 0,72 (IC de } 95 \% 0,5 \\
\text { Selon les données de } \\
7 \text { ERC }^{30-36} \text { portant sur } \\
851 \text { patients atteints d } \\
\text { SDRA }\end{array}$ \\
\hline $\begin{array}{l}\text { Durée du séjour } \\
\text { aux soins } \\
\text { intensifs }\end{array}$ & $\begin{array}{l}\text { Selon les données de } \\
3 \text { ERC }^{32-34} \text { portant sur } \\
297 \text { patients }\end{array}$ \\
\hline
\end{tabular}

Durée du séjour à Selon les données de l'hôpital $\quad 3 \mathrm{ERC}^{33,34,36}$ portant sur 324 patients

Selon les données de

Durée de la ventilation mécanique

Hyperglycémie
grave

Faiblesse neuromusculaire $6 \mathrm{ERC}^{30,31,33-36}$ portant sur 888 patients

RR 1,12 (IC de 95\% 1,01-1,24) Selon les données de 3 ERC $^{30,33,35}$ portant sur 565 patients

RR 0,85 (IC de $95 \%$ 0,62-1,18) Selon les données de 2 ERC $^{33,34}$ portant sur 271 patients

Saignements gastro-intestinaux RR 0,71 (IC de 95\% 0,30-1,73) Selon les données de 2 ERC $^{35,36}$ portant sur 250 patients

Surinfection

\author{
RR 0,82 (IC de 95\% 0,67-1,02) \\ Selon les données de \\ 5 ERC $^{30,33-36}$ portant sur \\ 798 patients
}

$\begin{array}{lc}\text { Risque de référence } & \text { Écart } \\ \text { du groupe témoin* } & \text { (IC de } 95 \% \text { ) }\end{array}$

$61,8 \%$ (IC de $95 \%$ )

\section{Qualité des données probantes}

Faible

(données très

indirectest)

Durée médiane du séjour aux soins intensifs : 8,0 jours

DM 0,1 jour $(-3,0$ à 3,2)

Durée médiane du séjour à l'hôpital : 18,0 jours

DM -3,6 jours

$(-7,2$ à $-0,02)$

Durée médiane de la
ventilation
mécanique:
14,5 jours

$67,6 \%$

$26,4 \%$

$14,0 \%$

$-4.0 \%$

$33,0 \%$

$$
\begin{gathered}
\text { DM -4,8 jours } \\
(-7,0 \text { à }-2,6)
\end{gathered}
$$

$8,1 \%$

(0,7\%-16,2\%)

$-3.9 \%$

$(-10 \%$ à $4.7 \%)$

\section{$-5.9 \%$}

(-9.8\% à $10.2 \%)$

in

(données très

indirectes, imprécision importante ${ }^{\star \star}$ )

Faible

(données très

indirectes, imprécision importante ${ }^{\star \star}$ )

$(-10.8 \%$ à $0.6 \%)$
Modérée

(données indirectes $† \dagger$ )

\section{Résumé en langage simple}

Les corticostéroïdes pourraient entraîner une diminution importante du risque de mortalité.

Les effets des corticostéroïdes sur la durée du séjour aux soins intensifs sont très incertains.

Les effets des corticostéroïdes sur la durée du séjour à l'hôpital sont très incertains.

Les corticostéroïdes pourraient réduire la durée de la ventilation mécanique.

Les corticostéroïdes pourraient augmenter le risque d'épisodes d'hyperglycémie grave. Les corticostéroïdes pourraient ne pas augmenter la faiblesse neuromusculaire.

Les corticostéroïdes pourraient ne pas augmenter le risque de saignements gastro-intestinaux.

Les corticostéroïdes n'augmentent probablement pas le risque de surinfection.

Remarque : COVID-19 = maladie à coronavirus 2019, DM = différence entre les moyennes, ERC = essai randomisé et contrôlé, GRADE = Grading of Recommendations Assessment, Development and Evaluation, IC = intervalle de confiance, $\mathrm{RR}=$ risque relatif, SDRA = syndrome de détresse respiratoire aiguë.

${ }^{\star}$ Risque de mortalité de référence pour les patients atteints de la COVID-19 et du SDRA n’ayant pas reçu de corticostéroïdes ${ }^{8}$. Les risques de référence pour la durée du séjour aux soins intensifs, la durée du séjour à l'hôpital, la durée de la ventilation mécanique et les préjudices ont été obtenus grâce à l'estimation de la médiane du groupe témoin dans les ERC inclus. †Deux niveaux ont été retirés en raison des données indirectes. La cause du SDRA n'est pas la même pour toutes les études et pourrait ne pas représenter la population atteinte de la COVID-19.

‡Deux niveaux ont été retirés en raison des données indirectes, un pour l'incohérence $\left(I^{2}=73 \%, p\right.$ pour l'hétérogénéité $\left.=0,03\right)$ et un pour l'imprécision parce que l'estimation des effets est cohérente avec les bénéfices et les préjudices.

$\S$ Deux niveaux ont été retirés en raison des données indirectes, et un pour imprécision en raison de l'IC incluant une réduction négligeable de la durée du séjour à l'hôpital. qUn niveau a été retiré en raison des données indirectes puisque nous ne nous attendons pas à ce que la population atteinte de la COVID-19 diffère beaucoup des autres populations pour les préjudices comme pour les bénéfices. Un autre niveau a été retiré pour l'imprécision en raison de la limite inférieure de l'IC, 0,7\% étant une augmentation négligeable du risque d'hyperglycémie.

**Un niveau a été retiré en raison des données indirectes puisque nous ne nous attendons pas à ce que la population atteinte de la CoVID-19 diffère beaucoup des autres populations pour les préjudices comme pour les bénéfices. Un autre niveau a été retiré pour imprécision puisque les estimations des effets sont cohérentes pour les bénéfices et les préjudices. ††Un niveau a été retiré en raison des données indirectes puisque nous ne nous attendons pas à ce que la population atteinte de la COVID-19 diffère beaucoup des autres populations pour les préjudices comme pour les bénéfices. Nous n'avons pas retiré de niveau pour imprécision parce que le plus important degré de méfait cohérent avec les données est de 7 pour 1000 , ce que nous considérons comme négligeable. 
Forme grave de COVID-19 : données indirectes d'essais randomisés sur des patients atteints d'une PEH

En tout, 13 ERC ${ }^{71-83}$ portant sur 2034 patients atteints d'une PEH se sont intéressés à plusieurs paramètres importants d'efficacité. Pour les patients atteints d'une PEH, en général, les données étaient de qualité élevée à faible. Après avoir retiré 2 niveaux en raison des données indirectes, toutes les données étaient donc de faible ou de très faible qualité. Les corticostéroïdes étaient associés à une réduction du risque de mortalité (risque relatif 0,70, IC de $95 \%$ 0,50-0,98, DM 3,1\% de moins), de la nécessité d'une ventilation mécanique ${ }^{72,75,76,79,82}$ (différence des risques [DR] 10,4\%, IC de $95 \%$ $4,3 \%-13,8 \%$ ), de la durée de la ventilation mécanique ${ }^{71,73,74,79,80}$ (DM 3,5 jours de moins, IC de 95\% 1,8-5,2 jours), de la durée du séjour aux soins intensifs ${ }^{72-76,78,79,82}$ et de la durée du séjour à l'hôpital 71-76,78,79,81,82,84 (tableau 7, figure 5). Une méta-analyse de $8 \mathrm{ERC}^{71,72,75,78,79,81,82,84}$ a montré que les corticostéroïdes pourraient

Tableau 3 : Résumé des conclusions et cote GRADE : effets des corticostéroïdes chez les patients atteints d'une forme grave de COVID-19, selon les données directes d'études d'observation sur des patients atteints de COVID-19 grave

\begin{tabular}{|c|c|c|c|c|c|}
\hline \multirow[b]{2}{*}{ Résultats } & \multirow[b]{2}{*}{ Effets relatifs } & \multicolumn{2}{|c|}{ Estimations des effets absolus } & \multirow[b]{2}{*}{$\begin{array}{c}\text { Qualité des données } \\
\text { probantes }\end{array}$} & \multirow[b]{2}{*}{$\begin{array}{c}\text { Résumé en langage } \\
\text { simple }\end{array}$} \\
\hline & & $\begin{array}{c}\text { Risque de } \\
\text { référence du } \\
\text { groupe témoin } \\
\text { (\%) }\end{array}$ & $\begin{array}{c}\text { Écart } \\
\text { (IC de } 95 \% \text { ) (\%) }\end{array}$ & & \\
\hline $\begin{array}{l}\text { Risque de } \\
\text { mortalité }\end{array}$ & $\begin{array}{l}\text { HR 2,30 (IC de } 95 \% \text { 1,00-5,29) } \\
\text { Selon les données de } 2 \text { études } \\
\text { d'observation }{ }^{19,23} \text { portant sur } \\
331 \text { patients atteints d'une } \\
\text { forme grave de COVID-19 }\end{array}$ & 10,4 & $11,9(0-33,7)$ & $\begin{array}{l}\text { Très faible } \\
\text { (imprécision } \\
\text { importantet) }\end{array}$ & $\begin{array}{l}\text { Les effets des } \\
\text { corticostéroïdes sur le } \\
\text { risque de mortalité sont } \\
\text { très incertains. }\end{array}$ \\
\hline
\end{tabular}

Tableau 4 : Résumé des conclusions et cote GRADE : effets des corticostéroïdes chez les patients atteints d'une forme grave de COVID-19, selon les données indirectes d'essais randomisés contrôlés et d'études d'observation sur des patients atteints du SRAS admis à l'hôpital

\begin{tabular}{|c|c|c|c|c|c|}
\hline \multirow[b]{2}{*}{ Résultats } & \multicolumn{3}{|c|}{ Estimations des effets absolus } & \multirow[b]{2}{*}{$\begin{array}{c}\text { Qualité des } \\
\text { données probantes }\end{array}$} & \multirow[b]{2}{*}{$\begin{array}{l}\text { Résumé en langage } \\
\text { simple }\end{array}$} \\
\hline & Effets relatifs & $\begin{array}{l}\text { Risque de } \\
\text { référence du } \\
\text { groupe témoin }\end{array}$ & $\begin{array}{c}\text { Écart } \\
\text { (IC de } 95 \% \text { ) }\end{array}$ & & \\
\hline $\begin{array}{l}\text { Risque de } \\
\text { mortalité }\end{array}$ & $\begin{array}{l}\text { HR 0,83 (IC de 95\% 0,41-1,66) } \\
\text { Selon les données de } \\
2 \text { études d'observation } 24,25 \\
\text { portant sur } 6129 \text { patients } \\
\text { atteints du SRAS }\end{array}$ & $10,4 \%$ * & $\begin{array}{c}-1.7 \% \\
(-6.0 \% \text { à } 6.3 \%)\end{array}$ & $\begin{array}{l}\text { Très faible } \\
\text { (données très } \\
\text { indirectes et } \\
\text { imprécision } \\
\text { importante†) }\end{array}$ & $\begin{array}{l}\text { Les effets des } \\
\text { corticostéroïdes sur le } \\
\text { risque de mortalité sont } \\
\text { très incertains. }\end{array}$ \\
\hline $\begin{array}{l}\text { Temps médian } \\
\text { après lequel } \\
\text { l'ARN du SRAS- } \\
\text { CoV devient } \\
\text { indétectable } \\
\text { dans le plasma }\end{array}$ & $\begin{array}{l}\text { Selon les données d'une ERC }{ }^{26} \\
\text { portant sur } 16 \text { patients }\end{array}$ & 8,0 days $\ddagger$ & $\begin{array}{l}\text { DM 4,0 jours } \\
(2,0-6,0)\end{array}$ & $\begin{array}{l}\text { Très faible } \\
\text { (risque de biais } \\
\text { important, données } \\
\text { très indirectes et } \\
\text { imprécisions } \\
\text { importantes§) }\end{array}$ & $\begin{array}{l}\text { Les effets des } \\
\text { corticostéroïdes sur le } \\
\text { temps après lequel l'ARN } \\
\text { du SRAS-CoV est } \\
\text { indétectable dans le } \\
\text { plasma sont très } \\
\text { incertains. }\end{array}$ \\
\hline \multicolumn{6}{|c|}{$\begin{array}{l}\text { Remarque : ARN = acide ribonucléique, COVID-19 = maladie à coronavirus 2019, DM = différence entre les moyennes, ERC = essai randomisé et contrôlé, GRADE = Grading of } \\
\text { Recommendations Assessment, Development and Evaluation, HR = rapport de risques (Hazard Ratio), IC = intervalle de confiance, SRAS = syndrome respiratoire aigu sévère, SRAS-CoV } \\
\text { coronavirus associé au SRAS. } \\
\text { `Risque de référence d'une étude sur des patients atteints d'une forme grave de COVID-19 n'ayant pas reçu de corticostéroïdes². } \\
\text { †Études d'observation aux données de faible qualité. Un niveau a été retiré en raison des données indirectes (nous avons appliqué les résultats aux patients atteints de la COVID-19, } \\
\text { mais les effets relatifs ont été dérivés des résultats de patients atteints du SRAS) et un pour imprécision importante (l'IC inclut un bienfait important et un méfait important). } \\
\text { łRisque de référence d'un ERC ayant rapporté le temps médian après lequel l'ARN du SRAS-CoV devient indétectable dans le plasma pour le groupe n'ayant pas reçu de } \\
\text { corticostéroïdes } \\
\text { §Essai randomisé et contrôlé aux données de qualité élevée. Des niveaux ont été retirés en raison du risque important de biais, des données indirectes (nous avons appliqué les } \\
\text { résultats aux patients atteints d'une forme grave de COVID-19, mais les effets relatifs ont été dérivés des résultats de patients atteints du SRAS) et d'une imprécision importante (en } \\
\text { raison de la petite taille de l'échantillon). }\end{array}$} \\
\hline
\end{tabular}




\begin{tabular}{|c|c|c|c|c|c|}
\hline \multirow[b]{2}{*}{ Résultats } & \multicolumn{3}{|c|}{ Estimations des effets absolus } & \multirow[b]{2}{*}{$\begin{array}{c}\text { Qualité des données } \\
\text { probantes }\end{array}$} & \multirow[b]{2}{*}{$\begin{array}{c}\text { Résumé en langage } \\
\text { simple }\end{array}$} \\
\hline & Effets relatifs & $\begin{array}{l}\text { Risque de } \\
\text { référence du } \\
\text { groupe témoin } \\
\text { (\%) }\end{array}$ & $\begin{array}{c}\text { Écart } \\
\text { (IC de } 95 \% \text { ) }\end{array}$ & & \\
\hline $\begin{array}{l}\text { Risque de } \\
\text { mortalité }\end{array}$ & $\begin{array}{l}\text { RC 0,75 (IC de } 95 \% \text { : 0,52-1,07) } \\
\text { Selon les données d'une étude } \\
\text { d'observation }{ }^{28} \text { portant sur } 290 \\
\text { patients atteints du SRMO }\end{array}$ & $10,4^{\star}$ & $\begin{array}{c}-2,4 \\
(-4,7 \text { à } 0,6)\end{array}$ & $\begin{array}{l}\text { Très faible } \\
\text { (données très indirectes } \\
\text { et imprécision } \\
\text { importante§) }\end{array}$ & $\begin{array}{l}\text { Les effets des } \\
\text { corticostéroïdes sur le } \\
\text { risque de mortalité sont } \\
\text { très incertains. }\end{array}$ \\
\hline $\begin{array}{l}\text { Clairance de } \\
\text { l'ARN du CoV } \\
\text { SRMO }\end{array}$ & $\begin{array}{l}\text { HR 0,35 (IC de } 95 \% \text { : } 0,17-0,72 \text { ) } \\
\text { Selon les données d'une étude } \\
\text { d'observation }{ }^{28} \text { portant sur } 189 \\
\text { patients atteints du SRMO }\end{array}$ & $29,8 \dagger$ & $\begin{array}{c}-18,2 \\
(-24,0 \text { à }-7,3)\end{array}$ & $\begin{array}{l}\text { Très faible } \\
\text { (données très indirectes } \\
\text { et imprécision } \\
\text { importanteฯ) }\end{array}$ & $\begin{array}{l}\text { Les effets des } \\
\text { corticostéroïdes sur le } \\
\text { délai de clairance de } \\
\text { l'ARN du CoV-SRMO sont } \\
\text { très incertains. }\end{array}$ \\
\hline \multicolumn{6}{|c|}{ 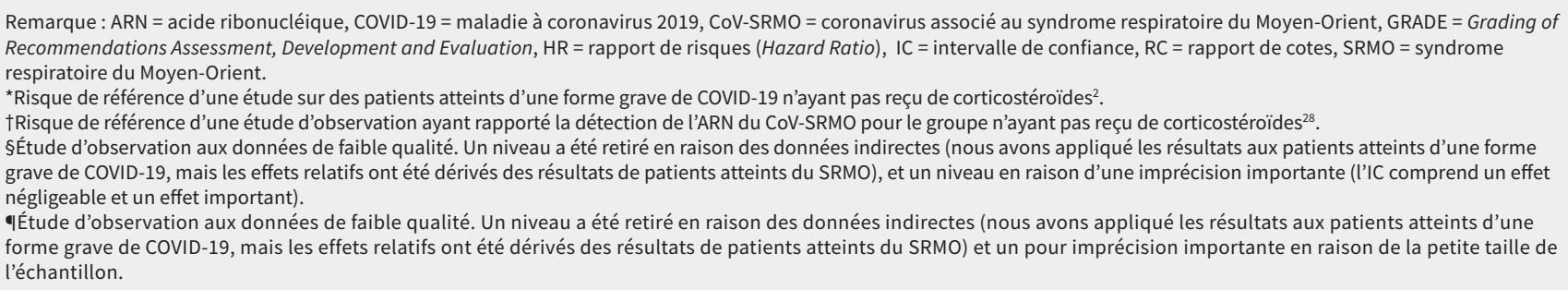 } \\
\hline
\end{tabular}

Tableau 6 : Résumé des conclusions et cote GRADE : effets des corticostéroïdes chez les patients atteints d'une forme grave de COVID-19, selon les données indirectes d'études d'observation sur des patients atteints d'influenza admis à l'hôpital

\begin{tabular}{|c|c|c|c|c|c|}
\hline \multirow[b]{2}{*}{ Résultats } & \multicolumn{3}{|c|}{ Estimations des effets absolus } & \multirow[b]{2}{*}{$\begin{array}{l}\text { Qualité des données } \\
\text { probantes }\end{array}$} & \multirow[b]{2}{*}{ Résumé en langage simple } \\
\hline & Effets relatifs & $\begin{array}{l}\text { Risque de } \\
\text { référence du } \\
\text { groupe } \\
\text { témoin }(\%)\end{array}$ & $\begin{array}{c}\text { Écart } \\
\text { (IC de } 95 \%) \\
(\%)\end{array}$ & & \\
\hline $\begin{array}{l}\text { Risque de } \\
\text { mortalité }\end{array}$ & $\begin{array}{l}\text { RC } 1,70 \text { (IC de } 95 \% \text { 1,31-2,21) } \\
\text { Selon les données de } 11 \text { études } \\
\text { d'observation }{ }^{43-45,47,52,55,57-59,61,68} \\
\text { portant sur } 8530 \text { participants }\end{array}$ & $10,4^{\star}$ & $\begin{array}{c}6,1 \\
(2,8-10,0)\end{array}$ & $\begin{array}{l}\text { Très faible } \\
\text { (données indirectes } \ddagger \text { ) }\end{array}$ & $\begin{array}{l}\text { Les effets des } \\
\text { corticostéroïdes sur le } \\
\text { risque de mortalité sont } \\
\text { très incertains. }\end{array}$ \\
\hline Surinfection & $\begin{array}{l}\text { RC } 2,74 \text { (IC de } 95 \% 1,51-4,95) \\
\text { Selon les données de } 7 \text { études } \\
\text { d'observation }{ }^{43,44,47,52,55,57,65} \\
\text { portant sur } 6114 \text { participants }\end{array}$ & $7,2 \dagger$ & $\begin{array}{c}10,3 \\
(3,3-20,5)\end{array}$ & $\begin{array}{l}\text { Très faible } \\
\text { (risque de biais } \\
\text { important et données } \\
\text { indirectes } \S \text { ) }\end{array}$ & $\begin{array}{l}\text { Les effets des } \\
\text { corticostéroïdes sur le } \\
\text { risque de surinfection sont } \\
\text { très incertains. }\end{array}$ \\
\hline $\begin{array}{l}\text { Ventilation } \\
\text { mécanique }\end{array}$ & $\begin{array}{l}\text { RC } 5,54 \text { (IC de } 95 \% 1,83-16,80 \text { ) } \\
\text { Selon les données de } 4 \text { études } \\
\text { d'observation }{ }^{52,57,59,61} \text { portant sur } \\
4364 \text { participants }\end{array}$ & $41,8 \S$ & $\begin{array}{c}38,1 \\
(15,0-50,6)\end{array}$ & $\begin{array}{l}\text { Très faible } \\
\text { (risque de biais } \\
\text { important et données } \\
\text { indirectest) }\end{array}$ & $\begin{array}{l}\text { Les effets des } \\
\text { corticostéroïdes sur la } \\
\text { nécessité d'une ventilation } \\
\text { mécanique sont très } \\
\text { incertains. }\end{array}$ \\
\hline \multicolumn{6}{|c|}{$\begin{array}{l}\text { Remarque : COVID-19 = maladie à coronavirus 2019, GRADE = Grading of Recommendations Assessment, Development and Evaluation, IC = intervalle de confiance, RC = rapport de } \\
\text { cotes. } \\
\text { “Risque de référence d'une étude sur des patients atteints d'une forme grave de COVID-19 n'ayant pas reçu de corticostéroïdes². } \\
\text { tRisque de référence de l'effet médian sur le groupe témoin pour les études incluses. } \\
\text { tÉtudes d'observation aux données de faible qualité. Les autres facteurs étaient les données indirectes (nous avons appliqué les résultats aux patients atteints d'une forme grave de } \\
\text { COVID-19, mais les effets relatifs ont été dérivés des résultats de patients atteints d'influenza admis à l'hôpital). } \\
\text { ફÉtudes d'observation aux données de faible qualité. Les autres facteurs étaient le risque élevé de biais de confusion en raison des estimations non ajustées et les données } \\
\text { indirectes (nous avons appliqué les résultats aux patients atteints d'une forme grave de COVID-19, mais les effets relatifs ont été dérivés des résultats de patients atteints } \\
\text { d'influenza admis à l'hôpital). }\end{array}$} \\
\hline
\end{tabular}


Tableau 7 : Résumé des conclusions et cote GRADE : effets des corticostéroïdes chez les patients atteints d'une forme grave de COVID-19, selon les données indirectes d'essais randomisés et contrôlés sur des patients atteints d'une pneumonie extrahospitalière admis à l'hôpital

\begin{tabular}{|c|c|c|c|c|c|}
\hline \multirow[b]{2}{*}{ Résultats } & \multirow[b]{2}{*}{ Effets relatifs } & \multicolumn{2}{|c|}{ Estimations des effets absolus } & \multirow[b]{2}{*}{$\begin{array}{l}\text { Qualité des données } \\
\text { probantes }\end{array}$} & \multirow[b]{2}{*}{$\begin{array}{c}\text { Résumé en langage } \\
\text { simple }\end{array}$} \\
\hline & & $\begin{array}{c}\text { Risque de } \\
\text { référence du } \\
\text { groupe témoin* }\end{array}$ & $\begin{array}{c}\text { Écart } \\
\text { (IC de } 95 \%)\end{array}$ & & \\
\hline Risque de mortalité & $\begin{array}{l}\text { RR 0,70 (IC de } 95 \% 0,50-0,98 \text { ) } \\
\text { Selon les données de } \\
13 \mathrm{ERC}^{71-83} \text { portant sur } \\
2034 \text { patients }\end{array}$ & $10,4 \%$ & $\begin{array}{c}-3.1 \% \\
(-0.2 \% \text { à }-5.2 \%)\end{array}$ & $\begin{array}{l}\text { Très faible } \\
\text { (données très indirectes† } \\
\text { et grande incohérence) }\end{array}$ & $\begin{array}{l}\text { Les effets des } \\
\text { corticostéroïdes sur le } \\
\text { risque de mortalité sont } \\
\text { très incertains. }\end{array}$ \\
\hline $\begin{array}{l}\text { Durée du séjour aux } \\
\text { soins intensifs }\end{array}$ & $\begin{array}{l}\text { Selon les données de } \\
8 \mathrm{ERC}^{72-76,78,79,82} \text { portant sur } \\
1376 \text { patients }\end{array}$ & $\begin{array}{l}\text { Durée médiane } \\
\text { du séjour aux } \\
\text { soins intensifs: } \\
8,3 \text { jours }\end{array}$ & $\begin{array}{l}\text { DM - } 1,7 \text { jour } \\
(-3,4 \text { à } 0,1)\end{array}$ & $\begin{array}{l}\text { Très faible } \\
\text { (grande incohérence, } \\
\text { données très indirectes } \\
\text { et imprécision } \\
\text { importanteł) }\end{array}$ & $\begin{array}{l}\text { Les effets des } \\
\text { corticostéroïdes sur la } \\
\text { durée du séjour aux } \\
\text { soins intensifs sont très } \\
\text { incertains. }\end{array}$ \\
\hline $\begin{array}{l}\text { Durée du séjour à } \\
\text { l'hôpital }\end{array}$ & $\begin{array}{l}\text { Selon les données de } \\
10 \mathrm{ERC}^{71-76,78,79,81,82,84} \text { portant } \\
\text { sur } 1636 \text { patients }\end{array}$ & $\begin{array}{l}\text { Durée médiane } \\
\text { du séjour à } \\
\text { l'hôpital : } \\
\text { 14,3 jours }\end{array}$ & $\begin{array}{l}\text { DM -1,8 jour } \\
(-2,8 \text { à }-0,8)\end{array}$ & $\begin{array}{l}\text { Très faible } \\
\text { (grande incohérence, } \\
\text { données très indirectes } \\
\text { et imprécision } \\
\text { importante§) }\end{array}$ & $\begin{array}{l}\text { Les effets des } \\
\text { corticostéroïdes sur la } \\
\text { durée du séjour à } \\
\text { l'hôpital sont très } \\
\text { incertains. }\end{array}$ \\
\hline $\begin{array}{l}\text { Nécessité d'une } \\
\text { ventilation mécanique }\end{array}$ & $\begin{array}{l}\text { RR: } 0,42 \text { (IC de } 95 \%: 0,230,76 \text { ) } \\
\text { Selon les données de } \\
5 \mathrm{ERC}^{72,75,76,79,82} \text { portant sur } \\
1017 \text { patients }\end{array}$ & $18,0 \%$ & $\begin{array}{c}-10,4 \% \\
(-13,8 \% \text { à }-4,3 \%)\end{array}$ & $\begin{array}{l}\text { Faible } \\
\text { (données très } \\
\text { indirectest) }\end{array}$ & $\begin{array}{l}\text { Les corticostéroïdes } \\
\text { pourraient réduire la } \\
\text { nécessité d'une } \\
\text { ventilation mécanique. }\end{array}$ \\
\hline $\begin{array}{l}\text { Durée de la ventilation } \\
\text { mécanique }\end{array}$ & $\begin{array}{l}\text { Selon les données de } \\
5 \mathrm{ERC}^{71,73,74,79,80} \text { portant sur } \\
199 \text { patients }\end{array}$ & $\begin{array}{l}\text { Durée médiane } \\
\text { de la ventilation } \\
\text { mécanique: } \\
11,3 \text { jours }\end{array}$ & $\begin{array}{c}\text { DM }-3,5 \text { jours } \\
(-5,2 \text { à }-1,8)\end{array}$ & $\begin{array}{l}\text { Très faible } \\
\text { (risque important de } \\
\text { biais et données très } \\
\text { indirectes } 1 \text { ) }\end{array}$ & $\begin{array}{l}\text { Les effets des } \\
\text { corticostéroïdes sur la } \\
\text { durée de la ventilation } \\
\text { mécanique sont très } \\
\text { incertains. }\end{array}$ \\
\hline Hyperglycémie grave & $\begin{array}{l}\text { RR } 1,62 \text { (IC de } 95 \% 1,02-2,67 \text { ) } \\
\text { Selon les données de } \\
8 \mathrm{ERC}^{71,72,75,78,79,81,82,84} \text { portant } \\
\text { sur } 1476 \text { patients }\end{array}$ & $9,2 \%$ & $\begin{array}{c}5,7 \% \\
(0,18 \% \text { à } 15,3 \%)\end{array}$ & $\begin{array}{l}\text { Faible } \\
\text { (données très indirectes } \\
\text { et imprécision } \\
\text { importante }^{\star \star} \text { ) }\end{array}$ & $\begin{array}{l}\text { Les corticostéroïdes } \\
\text { pourraient augmenter le } \\
\text { risque d'épisodes } \\
\text { d'hyperglycémie grave. }\end{array}$ \\
\hline $\begin{array}{l}\text { Saignements gastro- } \\
\text { intestinaux }\end{array}$ & $\begin{array}{l}\text { RR 0,99 (IC de } 95 \% 0,432,24 \text { ) } \\
\text { Selon les données de } \\
8 \mathrm{ERC}^{71-75,79,80,82} \text { portant sur } \\
1228 \text { patients }\end{array}$ & $3,0 \%$ & $\begin{array}{c}-0,03 \% \\
(-1,7 \% \text { à } 3,7 \%)\end{array}$ & $\begin{array}{l}\text { Faible } \\
\text { (données très indirectes } \\
\text { et imprécision } \\
\text { importante }^{\star \star} \text { ) }\end{array}$ & $\begin{array}{l}\text { Les corticostéroïdes } \\
\text { pourraient avoir peu ou } \\
\text { pas d'effet sur les } \\
\text { saignements gastro- } \\
\text { intestinaux. }\end{array}$ \\
\hline $\begin{array}{l}\text { Incidents } \\
\text { neuropsychiatriques }\end{array}$ & $\begin{array}{l}\text { RR } 1,91 \text { (IC de } 95 \% 0,685,39 \text { ) } \\
\text { Selon les données de } \\
4 \mathrm{ERC}^{72,81,82,84} \text { portant sur } \\
1142 \text { patients }\end{array}$ & $1,6 \%$ & $\begin{array}{c}1,4 \% \\
(-0,5 \% \text { à } 7 \%)\end{array}$ & $\begin{array}{l}\text { Faible } \\
\text { (données très indirectes } \\
\text { et imprécision } \\
\text { importanteฯ) }\end{array}$ & $\begin{array}{l}\text { Les corticostéroïdes } \\
\text { pourraient provoquer } \\
\text { une légère augmentation } \\
\text { des incidents } \\
\text { neuropsychiatriques. }\end{array}$ \\
\hline Superinfection & $\begin{array}{l}\text { RR } 1,31 \text { (IC de } 95 \% 0,692,50) \\
\text { Selon les données de } \\
8 \mathrm{ERC}^{71-74,78,81,82,84} \text { portant sur } \\
1500 \text { patients }\end{array}$ & $3,7 \%$ & $\begin{array}{c}1,1 \% \\
(-1,1 \% \text { à } 5,5 \%)\end{array}$ & $\begin{array}{l}\text { Faible } \\
\text { (données très indirectes } \\
\text { et imprécision } \\
\text { importanteq) }\end{array}$ & $\begin{array}{l}\text { Les corticostéroïdes } \\
\text { pourraient provoquer } \\
\text { une petite augmentation } \\
\text { ou aucune augmentation } \\
\text { des incidents de } \\
\text { surinfection. }\end{array}$ \\
\hline
\end{tabular}

Remarque : COVID-19 = maladie à coronavirus 2019, DM = différence entre les moyennes, $E R C=$ essai randomisé contrôlé, IC = intervalle de confiance, GRADE = Grading of Recommendations Assessment, Development and Evaluation, $\mathrm{PEH}=$ pneumonie extrahospitalière, $\mathrm{RR}$ = risque relatif.

*Risque de mortalité de référence pour des patients atteints de la COVID-19 et d'un SDRA n'ayant pas reçu de corticostéroïdes². Les risques de référence pour la durée du séjour aux soins intensifs, la durée du séjour à l'hôpital, la durée de la ventilation mécanique et les événements négatifs ont été obtenus à l'aide de la médiane des effets sur le groupe témoin dans les ERC inclus.

tDeux niveaux ont été retirés en raison des données indirectes. La cause de la pneumonie n'est pas la même pour toutes les études et pourrait ne pas être représentative de la population atteinte de la COVID-19. Un niveau a aussi été retiré pour les incohérences en raison d'un possible effet sur un sous-groupe qui suggère une diminution du risque de mortalité pour les personnes atteintes d'une pneumonie grave.

$\ddagger$ Deux niveaux ont été retirés en raison des données indirectes, un pour les incohérences $(/ 2=76 \%$, p pour l'hétérogénéité $=0,0001)$ et un pour l'imprécision en raison des estimations des effets qui sont cohérents pour les préjudices et les bénéfices importants.

$\S$ Deux niveaux ont été retirés en raison des données indirectes, un pour l'incohérence $\left(I^{2}=47 \%\right.$, p pour l'hétérogénéité $\left.=0,006\right)$ et un pour l'imprécision parce que la limite inférieure de l'IC comprend d'importants bénéfices et d'importants préjudices.

IUn niveau a été retiré en raison du risque de biais et deux niveaux en raison des données indirectes. Nous n'avons pas retiré de niveau en raison de l'incohérence : les estimations des effets allaient dans le même sens, malgré un $l^{2}$ de $54 \%$ et un $p$ de 0,07 .

**Un niveau a été retiré en raison des données indirectes, comme nous ne nous attendons pas à ce que la population atteinte de la COVID-19 diffère beaucoup des autres populations pour les bénéfices et les préjudices, et un niveau pour imprécision parce que les estimations des effets ne sont pas cohérentes avec les bénéfices et les préjudices. 
faire augmenter le risque d'hyperglycémie grave (DR 5,7\%, IC de $95 \%$ 0,18\%-15.3\%, données de qualité modérée pour la $\mathrm{PEH}$, données de faible qualité après ajustement en raison des données indirectes).

Les résultats suggèrent que les corticostéroïdes pourraient faire augmenter le risque de mortalité pour un sous-groupe selon la gravité de la pneumonie (forme grave de pneumonie, risque relatif 0,43 , IC de $95 \%$ 0,26-0,73; forme moins grave de pneumonie, risque relatif 1,00 , IC de $95 \% 0,64-1,56$; pour l'interaction, $p=0,02)$. Les différences sont toutefois habituellement relevées entre les études plutôt qu'au sein d'une même étude, différence qui provient en grande partie des résultats d'une petite étude ${ }^{73}$ ayant fait l'objet d'un arrêt précoce en raison de ce genre de bénéfices, et dont les données représentent presque certainement une grande surestimation des effets et n'apparaissent pour aucun autre paramètre. Ainsi l'effet sur le sous-groupe est peu crédible.

Pour les autres événements indésirables (incidents neuropsychiatriques $^{72,81,82,84}$, surinfection ${ }^{71-74,78,81,82,84}$ et saignements gastro-intestinaux ${ }^{71-75,79,80,82}$ ), pour la $\mathrm{PEH}$, les données étaient de qualité modérée pour des préjudices mineurs ou incertains, et de faible qualité après l'ajustement en raison des données indirectes (tableau 7 ).

\section{Interprétation}

Cette série de revues systématiques a guidé la rédaction d'une ligne directrice sur la prise en charge des patients atteints de la COVID-195. Les données directes d'une étude d'observation ${ }^{8}$ portant sur 84 patients atteints de la COVID-19 et d'un SDRA concordent avec les conclusions de notre revue systématique des ERC portant sur des patients atteints d'une maladie autre que la COVID-19, qui suggéraient que les corticostéroïdes pourraient réduire de plus de $15 \%$ le risque de mortalité chez les patients atteints de la COVID-19 et d'un SDRA et la durée de la ventilation mécanique. Les données semblent indiquer que les corticostéroïdes pourraient faire augmenter le risque d'hyperglycémie grave, bien qu'ils ne semblent avoir aucune incidence en matière d'autre effet indésirable important. Les données pour ces effets sont principalement de faible qualité.

Pour les patients atteints d'une forme grave de COVID-19, mais dont l'état n'est pas critique, les données directes de très faible qualité des études d'observation ont montré une augmentation du risque de mortalité avec la prise de corticostéroïdes. Pour le SRAS et le SRMO, les données observationnelles soulèvent la possibilité d'une réduction modeste du risque de mortalité associé à l'administration de corticostéroïdes, mais aussi une période plus importante avant la clairance du virus. Pour la $\mathrm{PEH}$, les données des ERC suggèrent aussi une réduction du risque de mortalité associée à l'administration de corticostéroïdes ainsi que d'autres bénéfices, notamment la réduction de la durée du séjour à l'hôpital et aux soins intensifs, de la nécessité d'une ventilation mécanique et de la durée de celleci. Des données de faible qualité suggèrent une augmentation probable de l'hyperglycémie et une possible augmentation mineure des incidents neuropsychiatriques et de surinfections, mais pas des saignements gastro-intestinaux. Les études d'observation sur l'influenza ont donné des résultats incohérents, soulevant la possibilité d'une augmentation marquée du risque de mortalité, du risque de surinfection et de la nécessité d'une ventilation mécanique associés à l'administration de corticostéroïdes.

Les forces de cette revue comprennent l'exhaustivité de la recherche, la sélection indépendante des études, l'abstraction des données, l'évaluation du risque de biais par 2 personnes et la présentation des effets absolus pour les paramètres dichotomiques. Nous avons classé la qualité des données selon l'approche GRADE, en portant attention aux enjeux méthodologiques majeurs, comme la différence dans les effets des données indirectes sur les préjudices et les bénéfices. Nous sommes plus hésitants quant à l'application des conclusions concernant les bénéfices constatés pour d'autres maladies aux patients atteints de la COVID-19, par rapport à l'application des conclusions concernant les préjudices. Pour les études d'observation, nous avons inclus, autant que possible, uniquement celles qui comportaient des analyses ajustées. Enfin, une force importante est la présentation d'une évaluation complète de toutes les données indirectes, notamment celles pour le SDRA, le SRAS, le SRMO, l'influenza et la PEH, dans un seul document.

Nous avons comparé notre revue à une autre revue systématique publiée portant sur l'administration de corticostéroïdes dans le traitement de la COVID-1985. Mis à part la COVID19, le SRAS et le SRMO, notre revue portait sur 3 autres populations : les patients atteints d'un SDRA, d'une PEH et de l'influenza. Notre revue est à jour jusqu'au 19 avril, et inclut des données publiées après la revue systématique qui était à jour au 15 mars $^{8,19-23}$. De plus, nous avons inclus, autant que possible, uniquement des études de cohorte et des études cas-témoins comportant des estimations des effets ajustées. Enfin, nous avons utilisé l'approche GRADE pour classer la qualité des données.

Pour le SDRA, notre revue a donné des résultats similaires à 1 autre revue systématique publiée ${ }^{29}$ qui comportait les dernières études publiées. Pour la $\mathrm{PEH}$, les résultats sur lesquels nous nous concentrons sont similaires à ceux d'autres revues récentes ${ }^{86-89}$ qui montraient que les corticostéroïdes pourraient réduire le risque de mortalité et la durée du séjour à l'hôpital, et faire augmenter le risque d'hyperglycémie.

Nos conclusions sur l'influenza concordent avec les autres revues systématiques précédentes ${ }^{90-92}$ qui montraient aussi une augmentation du risque de mortalité associée à l'administration de corticostéroïdes. Une revue ${ }^{90}$ portant uniquement sur les patients atteints d'une pneumonie d'origine grippale, excluant ceux atteints d'une forme modérée de la maladie ou aux soins intensifs, montre que les corticostéroïdes seraient associés à un plus grand risque de mortalité. À l'inverse, une autre revue ${ }^{92}$ portant sur les formes graves de l'influenza rapporte que, parmi les études ayant des estimations ajustées, les résultats ne montraient aucune différence statistiquement significative entre le groupe recevant des corticostéroïdes et le groupe témoin. 


\section{Limites de l'étude}

Les limites de cette étude sont essentiellement celles des données sous-jacentes, qui sont pour la plupart de faible qualité ou de très faible qualité pour les bénéfices. On pourrait nous reprocher de ne pas avoir élargi nos sources de données indirectes. Nous aurions pu, par exemple, inclure des données sur la pneumonie à Pneumocystis jiroveci, qui appuient l'administration de corticostéroïdes. Notre critère était l'inclusion de patients atteints d'une pneumonie d'origine virale dans les populations, ce qui est clairement le cas pour le SRAS, le SRMO et l'influenza, mais aussi pour le SDRA et la PEH.

De même, pour ce qui est des préjudices, la prise en compte des données provenant d'ERC sur l'administration à court terme de corticostéroïdes pour d'autres maladies pourrait avoir renforcé nos conclusions. Nous avons toutefois des données de qualité modérée pour les patients atteints du SDRA ne montrant aucune augmentation importante du risque de surinfection et des données de faible qualité sur l'augmentation de l'hyperglycémie grave. Des données de faible qualité suggèrent une possible augmentation mineure du nombre d'incidents neuropsychiatriques. Pour ce paramètre, les données d'autres maladies pourraient avoir été particulièrement utiles.

\section{Conclusion}

En raison du manque de données directes et des limites des données indirectes, il est essentiel pour les médecins et les chercheurs de coopérer à la réalisation d'études de grande qualité, particulièrement d'ERC rigoureux à grande échelle, pour évaluer les effets des corticostéroïdes chez les patients atteints de la COVID-19 et d'un SDRA et chez les patients atteints d'une forme grave de COVID-19 qui ne sont pas dans un état critique. Heureusement, des ERC, dont certaines qui portent sur le traitement par corticostéroïdes, sont en cours.

\section{Références}

1. WHO Director-General's opening remarks at the media briefing on COVID-1911 March 2020. Geneva: World Health Organization; 2020. Accessible ici : www. who.int/dg/speeches/detail/who-director-general-s-opening-remarks-at-the -media-briefing-on-covid-19---11-march-2020 (consulté le 23 avril 2020).

2. Guan WJ, Ni ZY, Hu Y, et al. Clinical characteristics of Coronavirus disease 2019 in China. N Engl J Med 2020;382:1708-1720.

3. Russell CD, Millar JE, Baillie JK. Clinical evidence does not support corticosteroid treatment for 2019-nCoV lung injury. Lancet 2020;395:473-5.

4. Shang L, Zhao J, Hu Y, et al. On the use of corticosteroids for 2019-nCoV pneumonia. Lancet 2020;395:683-4.

5. Ye Z, Rochwerg B, Wang Y, et al. Treatment of patients with nonsevere and severe coronavirus disease 2019: an evidence-based guideline. CMAJ 2020;192: E536-E545.

6. Clinical management of severe acute respiratory infection (SARI) when COVID-19 disease is suspected [interim guidance]. Geneva: World Health Organization; 13 mars 2020.

7. Moher D, Liberati A, Tetzlaff J, et al.; PRISMA Group. Preferred reporting items for systematic reviews and meta-analyses: the PRISMA statement. Ann Intern Med 2009;151:264-9, W64.

8. Wu C, Chen X, Cai Y, et al. Risk factors associated with acute respiratory distress syndrome and death in patients with Coronavirus disease 2019 pneumonia in Wuhan, China. JAMA Intern Med 2020; 13 mars [Cyberpublication avant impression]. doi: 10.1001/jamainternmed.2020.0994.

9. Whiting P, Savovic J, Higgins JP, et al. ROBIS: a new tool to assess risk of bias in systematic reviews was developed. J Clin Epidemiol 2016;69:225-34.
10. Guyatt GH, Busse JW. Modification of Cochrane Tool to assess risk of bias in randomized trials. Ottawa: Evidence Partners. Accessible ici : www.evidencepartners .com/resources/methodological-resources/ (consulté le 29 avril 2020).

11. Busse JW, Guyatt GH. Tool to assess risk of bias in cohort studies. Ottawa: Evidence Partners. Accessible ici : www.evidencepartners.com/resources/ methodological-resources/ (consulté le 29 avril 2020).

12. Busse JW, Guyatt GH. Tool to assess risk of bias in case-control studies. Ottawa: Evidence Partners. Accessible ici : www.evidencepartners.com/ resources/methodological-resources/ (consulté le 29 avril 2020).

13. Guyatt GH, Oxman AD, Vist GE, et al. GRADE: an emerging consensus on rating quality of evidence and strength of recommendations. BMJ 2008;336:924-6.

14. Guyatt GH, Oxman AD, Vist G, et al. GRADE guidelines: 4. Rating the quality of evidence-study limitations (risk of bias). J Clin Epidemiol 2011;64:407-15.

15. Guyatt GH, Oxman AD, Kunz R, et al. GRADE guidelines 6 . Rating the quality of evidence-imprecision. J Clin Epidemiol 2011;64:1283-93.

16. Guyatt GH, Oxman AD, Kunz R, et al. GRADE guidelines: 7. Rating the quality of evidence-inconsistency. J Clin Epidemiol 2011;64:1294-302.

17. Guyatt GH, Oxman AD, Kunz R, et al. GRADE guidelines: 8. Rating the quality of evidence-indirectness. J Clin Epidemiol 2011;64:1303-10.

18. Guyatt GH, Oxman AD, Montori V, et al. GRADE guidelines: 5 . Rating the quality of evidence-publication bias. J Clin Epidemiol 2011;64:1277-82.

19. Li X, Xu S, Yu M, et al. Risk factors for severity and mortality in adult COVID-19 inpatients in Wuhan. J Allergy Clin Immunol 2020; 12 avril [Cyberpublication avant impression]. pii: S0091-6749(20)30495-4. doi: 10.1016/j.jaci.2020.04.006.

20. Wang D, Wang J, Jiang Q, et al. No clear benefit to the use of corticosteroid as treatment in adult patients with Coronavirus disease 2019: a retrospective cohort study. medRxiv 24 avril 2020. doi: 10.1101/2020.04.21.20066258.

21. Xu K, Chen Y, Yuan J, et al. Factors associated with prolonged viral RNA shedding in patients with COVID-19. Clin Infect Dis 2020; 9 avril [Cyberpublication avant impression]. pii: ciaa351. doi: 10.1093/cid/ciaa351.

22. Yan D, Liu X, Zhu Y, et al. Factors associated with prolonged viral shedding and impact of lopinavir/ritonavir treatment in patients with SARS-CoV-2 infection. medRxiv 2020; 30 mars. doi: 10.1101/2020.03.22.20040832.

23. Lu X, Chen T, Wang Y, et al. Adjuvant corticosteroid therapy for critically ill patients with COVID-19. medRxiv 2020.

24. Lau EHY, Cowling BJ, Muller MP, et al. Effectiveness of Ribavirin and corticosteroids for severe acute respiratory syndrome. Am J Med 2009;122:1150.e11-.e21.

25. Long Y, Xu Y, Wang B, et al. Clinical recommendations from an observational study on MERS: glucocorticoids was benefit in treating SARS patients. Int J Clin Exp Med 2016;9:8865-73.

26. Lee N, Allen Chan KC, Hui DS, et al. Effects of early corticosteroid treatment on plasma SARS-associated coronavirus RNA concentrations in adult patients. $J$ Clin Virol 2004;31:304-9.

27. Alfaraj SH, Al-Tawfiq JA, Assiri AY, et al. Clinical predictors of mortality of Middle East respiratory syndrome coronavirus (MERS-CoV) infection: a cohort study. Travel Med Infect Dis 2019;29:48-50.

28. Arabi YM, Mandourah Y, Al-Hameed F, et al. Corticosteroid therapy for critically ill patients with Middle East respiratory syndrome. Am J Respir Crit Care Med 2018;197:757-67.

29. Lewis SR, Pritchard MW, Thomas CM, et al. Pharmacological agents for adults with acute respiratory distress syndrome. Cochrane Database Syst Rev 2019;7:CD004477.

30. Villar J, Ferrando C, Martinez D, et al. Dexamethasone treatment for the acute respiratory distress syndrome: a multicentre, randomised controlled trial. Lancet Respir Med 2020;8:267-76.

31. Abdelsalam Rezk N, Mohamed Ibrahim A. Effects of methyl prednisolone in early ARDS. Egyptian J Chest Dis Tuberc 2013;62:167-72.

32. Liu L, Li J, Huang YZ, et al. The effect of stress dose glucocorticoid on patients with acute respiratory distress syndrome combined with critical illness-related corticosteroid insuficiency. Zhonghua Nei Ke Za Zhi 2012;51:599-603.

33. Meduri GU, Golden E, Freire AX, et al. Methylprednisolone infusion in early severe ARDS: results of a randomized controlled trial. Chest 2007;131:954-63.

34. Steinberg KP, Hudson LD, Goodman RB, et al. Efficacy and safety of corticosteroids for persistent acute respiratory distress syndrome. N Engl J Med 2006;354:1671-84.

35. Tongyoo S, Permpikul C, Mongkolpun W, et al. Hydrocortisone treatment in early sepsis-associated acute respiratory distress syndrome: results of a randomized controlled trial. Crit Care 2016;20:329. 
36. Zhao WB, Wan S, Gu DF, et al. Therapeutic effect of glucocorticoid inhalation for pulmonary fibrosis in ARDS patients. Med J Chinese People's Liberation Army 2014;39:741-5.

37. Lansbury LE, Rodrigo C, Leonardi-Bee J, et al. Corticosteroids as adjunctive therapy in the treatment of influenza: an updated Cochrane systematic review and metaanalysis. Crit Care Med 2019; 15 novembre [Cyberpublication avant impression]. doi: 10.1097/CCM.0000000000004093.

38. Tsai M-J, Yang K-Y, Chan M-C, et al. Impact of corticosteroid treatment on clinical outcomes of influenza-associated ARDS: a nationwide multicenter study. Ann Intensive Care 2020;10:26.

39. Al-Busaidi M, Al Maamari K, Al'Adawi B, et al. Pandemic influenza A (H1N1) in Oman: epidemiology, clinical features, and outcome of patients admitted to Sultan Qaboos University Hospital in 2009. Oman Med J 2016;31:290-7.

40. Annane D. Pro: the illegitimate crusade against corticosteroids for severe H1N1 pneumonia. Am J Respir Crit Care Med 2011;183:1125-6.

41. Balaganesakumar SR, Murhekar MV, Swamy KK, et al. Risk factors associated with death among influenza A (H1N1) patients, Tamil Nadu, India, 2010. J Postgrad Med 2013;59:9-14

42. Boudreault AA, Xie H, Leisenring W, et al. Impact of corticosteroid treatment and antiviral therapy on clinical outcomes in hematopoietic cell transplant patients infected with influenza virus. Biol Blood Marrow Transplant 2011;17:979-86.

43. Brun-Buisson C, Richard J-CM, Mercat A, et al. Group R-SAHNvR. Early corticosteroids in severe influenza $\mathrm{A} / \mathrm{H} 1 \mathrm{~N} 1$ pneumonia and acute respiratory distress syndrome. Am J Respir Crit Care Med 2011;183:1200-6.

44. Cao B, Gao H, Zhou B, et al. Adjuvant corticosteroid treatment in adults with influenza A (H7N9) viral pneumonia. Crit Care Med 2016;44:e318-28.

45. Sheu C-C, Chang W-A, Tsai M-J, et al. Early corticosteroid treatment in patients with influenza-associated acute respiratory distress syndrome. Am J Respir Crit Care Med 2017;195:A2769.

46. Chawla R, Kansal S, Chauhan M, et al. Predictors of mortality and length of stay in hospitalized cases of 2009 influenza A (H1N1): experiences of a tertiary care center. Indian J Crit Care Med 2013;17:275-82.

47. Delaney JW, Pinto R, Long J, et al. The influence of corticosteroid treatment on the outcome of influenza A(H1N1pdm09)-related critical illness. Crit Care 2016;20:75.

48. Delgado-Rodríguez M, Castilla J, Godoy P, et al. Prognosis of hospitalized patients with 2009 H1N1 influenza in Spain: influence of neuraminidase inhibitors. J Antimicrob Chemother 2012;67:1739-45.

49. Han K, Ma H, An X, et al. Early use of glucocorticoids was a risk factor for critical disease and death from pH1N1 infection. Clin Infect Dis 2011;53:326-33.

50. Huang S-F, Fung C-P, Perng D-W, Wang F-D. Effects of corticosteroid and neuraminidase inhibitors on survival in patients with respiratory distress induced by influenza virus. J Microbiol Immunol Infect 2017;50:586-94.

51. Jain S, Kamimoto L, Bramley AM, et al. Hospitalized patients with 2009 H1N1 influenza in the United States, April-June 2009. N Engl J Med 2009;361:1935-44.

52. Kim S-H, Hong S-B, Yun S-C, et al. Corticosteroid treatment in critically ill patients with pandemic influenza A/H1N1 2009 infection: analytic strategy using propensity scores. Am J Respir Crit Care Med 2011;183:1207-14.

53. Kinikar AA, Kulkarni RK, Valvi CT, et al. Predictors of mortality in hospitalized children with pandemic H1N1 influenza 2009 in Pune, India. Indian J Pediatr 2012;79:459-66.

54. Kudo K, Takasaki J, Manabe T, et al. Systemic corticosteroids and early administration of antiviral agents for pneumonia with acute wheezing due to influenza A(H1N1)pdm09 in Japan. PLoS One 2012;7:e32280.

55. Lee N, Leo Y-S, Cao B, et al. Neuraminidase inhibitors, superinfection and corticosteroids affect survival of influenza patients. Eur Respir J 2015;45:1642-52.

56. Li F, Chen G, Wang J, et al. A case-control study on risk factors associated with death in pregnant women with severe pandemic H1N1 infection. BMJ Open 2012;2. pii: e000827.

57. Li H, Yang S-G, Gu L, et al. Effect of low-to-moderate-dose corticosteroids on mortality of hospitalized adolescents and adults with influenza A(H1N1)pdm09 viral pneumonia. Influenza Other Respir Viruses 2017;11:345-54.

58. Liem NT, Tung CV, Hien ND, et al. Clinical features of human influenza A (H5N1) infection in Vietnam: 2004-2006. Clin Infect Dis 2009;48:1639-46.

59. Linko R, Pettilä V, Ruokonen E, et al. Corticosteroid therapy in intensive care unit patients with PCR-confirmed influenza A(H1N1) infection in Finland. Acta Anaesthesiol Scand 2011;55:971-9.
60. Mady A, Ramadan OS, Yousef A, et al. Clinical experience with severe $2009 \mathrm{H} 1 \mathrm{~N} 1$ influenza in the intensive care unit at King Saud Medical City, Saudi Arabia. J Infect Public Health 2012;5:52-6.

61. Moreno G, Rodríguez A, Reyes LF, et al. Corticosteroid treatment in critically ill patients with severe influenza pneumonia: a propensity score matching study. Intensive Care Med 2018;44:1470-82.

62. Ono S, Ono Y, Matsui $\mathrm{H}$, et al. Factors associated with hospitalization for seasonal influenza in a Japanese nonelderly cohort. BMC Public Health 2016;16:922.

63. Patel KK, Patel AK, Mehta PM, et al. Clinical outcome of novel H1N1 (swine flu)infected patients during 2009 pandemic at tertiary referral hospital in Western India. J Glob Infect Dis 2013;5:93-7.

64. Sertogullarindan B, Ozbay B, Gunini H, et al. Clinical and prognostic features of patients with pandemic 2009 influenza $A(\mathrm{H} 1 \mathrm{~N} 1)$ virus in the intensive care unit. Afr Health Sci 2011;11:163-70.

65. Viasus D, Paño-Pardo JR, Cordero E, et al. Effect of immunomodulatory therapies in patients with pandemic influenza A (H1N1) 2009 complicated by pneumonia. $J$ Infect 2011;62:193-9.

66. Wirz SA, Blum CA, Schuetz $P$, et al. Pathogen- and antibiotic-specific effects of prednisone in community-acquired pneumonia. Eur Respir J 2016;48:1150-9.

67. Wu U-I, Wang J-T, Ho Y-C, et al. Factors associated with development of complications among adults with influenza: a 3-year prospective analysis. J Formos Med Assoc 2012;111:364-9.

68. Xi X, Xu Y, Jiang L, et al. Hospitalized adult patients with 2009 influenza $A(H 1 N 1)$ in Beijing, China: risk factors for hospital mortality. BMC Infect Dis 2010;10:256.

69. Yu H, Yang Y, Zhang Q. Clinical characteristics and risk factors of severe patients with novel pandemic influenza A H1N1. Chinese J Integr Tradit West Med Intentive Crit Care 2011;18:142-5.

70. Siemieniuk RA, Meade MO, Alonso-Coello P, et al. Corticosteroid therapy for patients hospitalized with community-acquired pneumonia: a systematic review and meta-analysis. Ann Intern Med 2015;163:519-28.

71. Gang LI, Chengdong GU, Zhang S, et al. Value of glucocorticoid steroids in the treatment of patients with severe community-acquired pneumonia complicated with septic shock. Chinese Crit Care Med 2016;28:780-4.

72. Blum CA, Nigro N, Briel M, et al. Adjunct prednisone therapy for patients with community-acquired pneumonia: a multicentre, double-blind, randomised, placebo-controlled trial. Lancet 2015;385:1511-8.

73. Confalonieri M, Urbino R, Potena A, et al. Hydrocortisone infusion for severe community-acquired pneumonia: a preliminary randomized study. Am J Respir Crit Care Med 2005;171:242-8.

74. El-Ghamrawy AH, Shokeir MH, Esmat AA. Effects of low-dose hydrocortisone in ICU patients with severe community-acquired pneumonia. Egyptian J Chest Dis Tuberc 2006;55:91-9.

75. Fernández-Serrano S, Dorca J, Garcia-Vidal C, et al. Effect of corticosteroids on the clinical course of community-acquired pneumonia: a randomized controlled trial. Crit Care 2011;15:R96.

76. Marik $\mathrm{P}$, Kraus $\mathrm{P}$, Sribante J, et al. Hydrocortisone and tumor necrosis factor in severe community-acquired pneumonia: a randomized controlled study. Chest 1993;104:389-92.

77. McHardy VUSM. Ampicillin dosage and use of prednisolone in treatment of pneumonia: co-operative controlled trial. BMJ 1972;4:569-73.

78. Meijvis SC, Hardeman $\mathrm{H}$, Remmelts $\mathrm{HH}$, et al. Dexamethasone and length of hospital stay in patients with community-acquired pneumonia: a randomised, double-blind, placebo-controlled trial. Lancet 2011;377:2023-30.

79. Nafae RM, Ragab MI, Amany FM, et al. Adjuvant role of corticosteroids in the treatment of community-acquired pneumonia. Egyptian J Chest Dis Tuberc 2013;62:439-45.

80. Sabry NA, Omar E-D. Corticosteroids and ICU course of community-acquired pneumonia in Egyptian settings. Pharmacol Pharmacy 2011;2:73-81.

81. Snijders D, Daniels JM, de Graaff CS, et al. Efficacy of corticosteroids in community-acquired pneumonia: a randomized double-blinded clinical trial. Am J Respir Crit Care Med 2010;181:975-82.

82. Torres A, Sibila O, Ferrer M, et al. Effect of corticosteroids on treatment failure among hospitalized patients with severe community-acquired pneumonia and high inflammatory response: a randomized clinical trial. JAMA 2015;313:677-86.

83. Wagner HN Jr, Bennett I Jr, Lasagna L, et al. The effect of hydrocortisone upon the course of pneumococcal pneumonia treated with penicillin. Bull Johns Hopkins Hosp 1956;98:197-215. 
84. Mikami K, Suzuki M, Kitagawa H, et al. Efficacy of corticosteroids in the treatment of community-acquired pneumonia requiring hospitalization. Lung 2007;185:249-55.

85. Yang Z, Liu J, Zhou Y, et al. The effect of corticosteroid treatment on patients with coronavirus infection: a systematic review and meta-analysis. J Infect 2020; 10 avril [Cyberpublication avant impression]. pii: S0163-4453(20)30191-2. doi: 10.1016/j. jinf.2020.03.062.

86. Huang J, Guo J, Li H, et al. Efficacy and safety of adjunctive corticosteroids therapy for patients with severe community-acquired pneumonia: A systematic review and meta-analysis. Medicine (Baltimore) 2019;98:e14636.

87. Jiang S, Liu T, Hu Y, et al. Efficacy and safety of glucocorticoids in the treatment of severe community-acquired pneumonia: a meta-analysis. Medicine (Baltimore) 2019;98:e16239.
88. Stern A, Skalsky K, Avni T, et al. Corticosteroids for pneumonia. Cochrane Database Syst Rev 2017;(12):CD007720.

89. Wu WF, Fang Q, He GJ. Efficacy of corticosteroid treatment for severe communityacquired pneumonia: a meta-analysis. Am J Emerg Med 2018;36:179-84.

90. Ni Y-N, Chen G, Sun J, et al. The effect of corticosteroids on mortality of patients with influenza pneumonia: a systematic review and meta-analysis. Crit Care 2019;23:99.

91. Zhang Y, Sun W, Svendsen ER, et al. Do corticosteroids reduce the mortality of influenza A (H1N1) infection? A meta-analysis. Crit Care 2015;19:46.

92. Zhou Y, Fu X, Liu X, et al. Use of corticosteroids in influenza-associated acute respiratory distress syndrome and severe pneumonia: a systemic review and meta-analysis. Sci Rep 2020;10:3044.
Intérêts concurrents : Bram Rochwerg est un chercheur participant à un essai financé par une subvention des Instituts de recherche en santé du Canada qui évalue les effets des corticostéroïdes chez les patients atteints de la COVID-19. Aucun autre intérêt concurrent déclaré.

Cet article a été révisé par des pairs.

Affiliations : Département de Health Research Methods, Evidence and Impact (Ye, Tangamornsuksan, Rochwerg, Guyatt, Colunga-Lozano, Yao, Motaghi, Fang, Xiao), Université McMaster, Hamilton, Ont.; département de pharmacie (Wang), hôpital de Chaoyang à Beijing, Capital Medical University, Beijing (Chine); département de médecine clinique (Colunga-Lozano), centre des sciences de la santé, université de Guadalajara, Guadalajara (Mexique); département de médecine Communautaire (Prasad), North DMC Medical College, New Delhi (Inde); Faculté de médecine et de santé publique (Tangamornsuksan), HRH Princess Chulabhorn College of Medical Science, Chulabhorn Royal Academy, Bangkok (Thaïlande); département de médecine (Rochwerg); DeGroote Institute for Pain Research and Care (Couban), Université McMaster, Hamilton, Ont.; département de pharmacie clinique (Ghadimi), Faculté de pharmacie, Tehran University of Medical Sciences, Téhéran (Iran); chaire d'épidémiologie et de médecine préventive (Bala), École de médecine de l'Université Jagellonne, Cracovie (Pologne); département de biostatistique (Gomaa), High institute of Public Health, Alexandria University, Alexandrie (Égypte); Centre d'information sur les médicaments (Gomaa), Tanta Chest Hospital, ministère de la Santé et des Populations, Égypte; Clinical Medicine College of Acupuncture, Moxibustion and Rehabilitation (Fang),
Guangzhou University of Chinese Medicine, Guangdong (Chine); West China School of Nursing (Xiao), West China Hospital, Sichuan University (Chine)

Collaborateurs : Zhikang Ye et Gordon Guyatt ont contribué à la conception des travaux. Zhikang Ye, Ying Wang, Luis Enrique Colunga-Lozano, Manya Prasad et Gordon Guyatt ont contribué à l'élaboration des travaux. Rachel Couban, Zhikang Ye, Ying Wang, Luis Enrique Colunga-Lozano, Manya Prasad, Wimonchat Tangamornsuksan, Liang Yao, Shahrzad Motaghi, Maryam Ghadimi, Malgorzata Bala, Huda Gomaa, Fang et Yingqi Xiao ont contribué à la collecte, l'analyse et l'interprétation des données. Zhikang Ye, Ying Wang, Luis Enrique Colunga-Lozano et Manya Prasad ont participé à la rédaction du manuscrit. Tous les auteurs ont révisé de façon critique le contenu intellectuel important, ont donné leur approbation finale pour la version destinée à être publiée et assument l'entière responsabilité de tous les aspects du travail. Zhikang Ye, Ying Wang, Luis Enrique Colunga-Lozano et Manya Prasad sont les auteurs principaux.

\section{Financement : Aucun.}

Partage des données : Les données extraites des études incluses sont présentées à la section Résultats; toutefois, les tableaux complets de données extraites sont disponibles auprès de l'auteur-ressource.

Remerciements : Quazi Ibrahim, Lehana Thabane, Diane Heels-Ansdell, Jason Busse et Li Wang ont offert de l'aide et des suggestions pour l'analyse statistique. Yingqi Xiao bénéficie de l'aide du Conseil des bourses de Chine.

Accepté : $1^{\text {er }}$ mai 2020

Correspondance : Gordon Guyatt, guyatt@mcmaster.ca 